BUTP-97/19

hep-lat/9708005

\title{
Quenched Chiral Perturbation Theory to one loop
}

\author{
Gilberto Colangelo ${ }^{a, \text { 田 }}$ and Elisabetta Pallante ${ }^{b, q}$ \\ ${ }^{a}$ INFN - Laboratori Nazionali di Frascati, \\ P.O. Box 13, I-00044 Frascati (Rome), Italy \\ ${ }^{b}$ University of Bern, Sidlerstrasse 5, \\ CH-3012 Bern, Switzerland
}

\begin{abstract}
We calculate the divergences of the generating functional of quenched Chiral Perturbation Theory at one loop, and renormalize the theory by an appropriate definition of the counterterms. We show that the quenched chiral logarithms can be accounted for by defining a renormalized $B_{0}$ parameter which, at lowest order, is proportional to the vacuum expectation value of the scalar quark density. Finally, we calculate several quantities at one loop to better analyze the modifications induced by quenching in the ultraviolet finite part of the one-loop corrections. We point out that some of the finite loop corrections may diverge in the chiral limit.
\end{abstract}

PACS: 12.39.Fe, 12.38.Gc.

Keywords: Chiral Perturbation Theory, Quenched Approximation, Lattice QCD.

August 1997

\footnotetext{
${ }^{1} \mathrm{e}-$ mail: colangelo@lnf.infn.it

${ }^{2}$ e-mail: pallante@butp.unibe.ch
} 


\section{Introduction}

Most lattice calculations of QCD in its non-perturbative regime and weak interactions use at present the quenched approximation, i.e. neglect the effect of virtual quark loops. Taking them into account considerably increases computing times. This means that presumably the quenched approximation will remain with us for quite a long time: even with computers much faster than those presently available, it will always offer the chance to make a low cost exploratory calculation before embarking on a full QCD simulation.

Simulations of quenched QCD would be much more useful if we had a real understanding of the effects of this approximation. Investigations in this direction have been made by several authors [1, 2, 3]. At present we see one main approach that has proven to be the most systematic, and also to incorporate most of the useful ideas that have been proposed on the subject. This method is called quenched Chiral Perturbation Theory ( $\mathrm{qCHPT}$ ), and has been originally proposed by Bernard and Golterman in Ref. [3] for the purely strong sector (strong interactions in the presence of external fields). It has been recently extended to the heavy-light meson sector [ [4, to vector mesons [5] and to the baryon sector [6]. It has been also used in the context of non-leptonic weak interactions [2, 7].

Let us shortly review the main ideas behind this approach. The difficulty to control the quenched approximation comes from the fact that one is modifying the theory at the non-perturbative level. On the other hand we know that at low energy it is possible to define a perturbative scheme to study the strong interactions: this scheme is known as Chiral Perturbation Theory (CHPT). In this framework the expansion parameter is given by the energy of the weakly interacting Goldstone bosons of the spontaneously broken chiral symmetry: these have a vanishing interaction at zero energy, as symmetry dictates. The chiral symmetry imposes also a set of relations between the coefficients of this expansion in different amplitudes. Those relations do not fully constrain the theory that at each order of the expansion has a number of free constants. These constants incorporate the effect of the non-perturbative QCD dynamics. Under the assumption that in the quenched approximation the mechanism of spontaneous chiral symmetry breaking is preserved, one may attempt to construct a perturbative scheme for the quenched case, analogous to the one valid in the full QCD case. In this manner one would be able to calculate those effects of quenching that modify the perturbative, calculable part of the theory. On the other hand, the changes in the unconstrained low energy constants remain unknown, being due to the modifications which affect the non-perturbative QCD dynamics. This method has the advantage of introducing from the start this clear, useful separation between the non-perturbative dynamics of the fundamental theory and the perturbative, predictable dynamics of the Goldstone bosons.

A peculiar aspect of the quenched approximation comes from the $U(1)$ axial anomaly of QCD. In the fundamental theory the would-be-Goldstone boson (the 
$\eta^{\prime}$ ) does not become massless in the chiral limit, since the axial anomaly generates a singlet component (heavy) mass at the level of the effective theory. Thus, in the real world the $\eta^{\prime}$ is heavy and decoupled from the octet of the pseudo-Goldstone bosons. In the quenched approximation this decoupling stops halfway: only one of the diagrams that are responsible for the decoupling of the $\eta^{\prime}$ survives. At the level of the effective theory this has important consequences: the singlet field remains light (degenerate with the Goldstone bosons) and has to be treated on the same footing as the octet fields. However its two-point function develops a double pole and does not admit an interpretation as a propagator. Treating the singlet as a dynamical degree of freedom brings in new constants in the effective theory. One of them is a new mass scale (the singlet mass $m_{0}$ ) that is generated by the anomaly, and that does not vanish in the chiral limit. This mass appears in the numerator of the double-pole term in the singlet two-point function. As different authors have shown [2, 3], this double pole is responsible for the presence of a new type of chiral logarithms (we denote them as quenched chiral $\operatorname{logs}$ ) in loop corrections, of the form $m_{0}^{2} \ln M_{\pi}^{2}$, as opposed to the standard ones $M_{\pi}^{2} \ln M_{\pi}^{2}$. This is one of the main qualitative differences that arises from the quenched version of CHPT.

So far, works in quenched CHPT have concentrated on specific processes, analyzing the changes induced in Goldstone boson loops and the size of the effect of quenched chiral logarithms. The aim of the present work is to perform a complete renormalization of the theory at the one-loop level, on the same line as what has been done by Gasser and Leutwyler in the ordinary CHPT case [8, 9]. This requires a calculation of all the ultraviolet divergent pieces of the generating functional and a definition of the Lagrangian at order $p^{4}$, the next-to-leading order. The advantages of the present analysis are the following:

1) The calculation of the divergences and renormalization can be done for a generic number of flavours $N$. As we have shown in Ref. [10] the $N-$ dependence of the divergences can be used to verify the cancellation of quark loops in the effective theory.

2) Like in the standard case, the calculation of the divergences at the generating functional level provides a useful check for single amplitude calculations. This check is even more welcome in qCHPT where the number of graphs to be computed becomes soon very large.

3) This calculation allows to have full control on the divergences due to singlet loops. In particular we will show that quenched chiral logarithms can be accounted for via a renormalization of the low-energy constant $B_{0}$ (which is proportional to the $\bar{q} q$ condensate). This constant appears in all other quantities through the pion mass squared, with the only exception of $\bar{q} q$ matrix elements, that have it as an explicit factor. 
After having performed the one-loop renormalization, we will devote our attention to the ultraviolet finite part of the one-loop corrections, by computing specific physical quantities at one loop. The relevance of the finite part of the loop corrections is in the fact that they may contain terms which diverge in the chiral limit like an inverse power of the quark mass. One can realize that this may happen by simply looking at the standard chiral power counting [11, and taking into account the fact that in quenched CHPT a new vertex appears with chiral order zero (the vertex proportional to $m_{0}^{2}$ ). Power-like chiral divergences and quenched chiral logs are the crucial problem of the quenched version of CHPT: the effective theory is defined as an expansion around the chiral limit, and this limit is no more well defined in the quenched case. On the other hand these divergences seem to be unavoidable in the present framework and it looks plausible that they are a direct consequence of the sicknesses of quenched QCD. To clarify this very important point, a direct evidence of these effects in lattice simulations of quenched QCD would be most welcome.

In our analysis of various observables we will give the complete one-loop results. Our aim is not just to make predictions, or to compare with numbers produced in lattice simulations. Rather, we would like to show in detail how the quenched approximation distorts the matrix elements. For this reason we will only work in Minkowski space-time: all the formulae will be given with the idea that one should be able to easily see the difference from the corresponding ones calculated in standard CHPT. In particular we will stress the presence of terms divergent in the chiral limit and of unphysical threshold singularities in Minkowski space-time at infinite volume. These type of singularities have been already discussed in the literature [12, 18], and have lead to the conclusion that quenched CHPT makes sense only in Euclidean space-time. Despite this, we still prefer to calculate amplitudes in Minkowski space-time, considering them as formal expressions. As we just said this will make the comparison to standard CHPT amplitudes easier; on the other hand, the modifications needed to go to Euclidean space-time can be easily implemented.

The plan of the paper is as follows. In Section 2 we outline the main steps from CHPT to its quenched version. We give the leading order Lagrangian and define our notation, both for CHPT and quenched CHPT. In Section 3 we calculate the divergences of qCHPT to one loop using the background field method, while Section 4 contains the list of counterterms for a generic number of flavours $N$ and for $N=3$ and 2. This completes the renormalization of the theory at the one-loop level. In Section 5 we analyze a few quantities to one loop in the two degenerate flavours case. These are the $\bar{q} q$ condensate, the pion mass and decay constant, the scalar and vector form factors of the pion, and the $\pi \pi$ scattering amplitude. In Section 6 we state our conclusions. We have also three appendices. In Appendix A we give a simple derivation of the divergent term proportional to $m_{0}^{2}$ in the quenched generating functional. In Appendix B we give the explicit $N$-dependence of the divergences in the non-leptonic weak interactions sector, 
and guess the divergences in the quenched case by simply dropping any $N$ dependence. Finally, in Appendix $\mathrm{C}$ we give the explicit expressions for the one-loop functions which enter the calculations.

\section{From CHPT to its quenched version}

In this section we introduce the standard notation of Chiral Perturbation Theory, that will be also used in its quenched version. We work in Minkowski space-time in both cases for ease of comparison. For any further detail in the derivation of the CHPT Lagrangian we refer the reader to the original works by Gasser and Leutwyler [8, 9]. The construction of the CHPT Lagrangian is based on the identification of the symmetry group of the QCD Lagrangian in the chiral limit, which, for $N$ flavours is given by $U(N)_{L} \otimes U(N)_{R}$, and on the well supported assumption that the symmetry of the subgroup $S U(N)_{L} \otimes S U(N)_{R}$ is spontaneously broken to $S U(N)_{V}$. The extension of this construction to the quenched case was proposed by Bernard and Golterman [3] on the basis of an observation made by Morel [1]. He observed that, formally, a Lagrangian corresponding to quenched QCD can be obtained by adding to the QCD Lagrangian a term which is totally analogous to that for quark fields, but which contains ghost spin- $1 / 2$ fields with wrong, i.e. bosonic statistics. The symmetry of the resulting Lagrangian in the chiral limit is larger than that of QCD and is given by the graded group: $U(N \mid N)_{L} \otimes U(N \mid N)_{R}$, describing transformations between $N$ physical flavours and $N$ ghost flavours. It is then assumed that also in the quenched case a spontaneous symmetry breaking down to the diagonal subgroup $S U(N \mid N)_{V}$ occurs. Like in standard QCD, the $U(1)_{A}$ symmetry is anomalous.

\subsection{Standard CHPT}

Chiral Perturbation Theory describes the dynamics of the octet Goldstone bosons fields (pions) of the spontaneously broken chiral symmetry of QCD. It is an expansion in powers of the energy of the Goldstone bosons and the light quark masses. The lowest order CHPT Lagrangian, i.e. at order $p^{2}$ and linear in the quark masses, can be written in the following form:

$$
\mathcal{L}_{2}=\frac{F^{2}}{4}\left\langle D_{\mu} U D^{\mu} U^{\dagger}+U^{\dagger} \chi+\chi^{\dagger} U\right\rangle=\frac{F^{2}}{4}\left\langle u_{\mu} u^{\mu}+\chi_{+}\right\rangle,
$$

where $\langle\ldots\rangle$ stands for the trace over flavour indices, $F$ is the bare pion decay constant and the fields are defined as follows

$$
\begin{aligned}
U=u^{2} & =\exp (\sqrt{2} i \phi / F) \\
D_{\mu} U & =\partial_{\mu} U-i r_{\mu} U+i U l_{\mu}, \\
\chi & =2 B_{0}(s+i p)
\end{aligned}
$$




$$
\begin{aligned}
& u_{\mu}=i u^{\dagger} D_{\mu} U u^{\dagger}=u_{\mu}^{\dagger}, \\
& \chi_{+}=u \chi^{\dagger} u+u^{\dagger} \chi u^{\dagger} .
\end{aligned}
$$

The Lagrangian contains the external sources $s, p, v_{\mu}, a_{\mu}, r_{\mu}=v_{\mu}+a_{\mu}, l_{\mu}=$ $v_{\mu}-a_{\mu}$, which are $N \times N$ matrices, with $N$ the number of flavours. The field $\phi$ is an $N \times N$ matrix that contains the Goldstone bosons fields: $\phi=1 / \sqrt{2} \sum_{i=1}^{N^{2}-1} \lambda_{i} \phi^{i}$. In case, one may add to $\phi$ a singlet component, so that $\langle\phi\rangle=\phi_{0}$. In the presence of a singlet component the Lagrangian in Eq. (2.1) is invariant under $U(N)_{L} \otimes$ $U(N)_{R}$. Since in QCD the $U(1)_{A}$ subgroup is anomalous the breaking pattern $U(N)_{L} \otimes U(N)_{R} \rightarrow S U(N)_{L} \otimes S U(N)_{R} \otimes U(1)_{V}$ is realized. The invariance under the residual unbroken group allows for the presence of extra functions of the singlet component $\phi_{0}$ only. A possible choice for this Lagrangian, compatible with $P, C, T$ and chiral invariance, is (see also [9] for a different choice):

$$
\mathcal{L}_{2}=V_{1}\left(\phi_{0}\right)\left\langle D_{\mu} U D^{\mu} U^{\dagger}\right\rangle+V_{2}\left(\phi_{0}\right)\left\langle U^{\dagger} \chi+\chi^{\dagger} U\right\rangle-V_{0}\left(\phi_{0}\right)+V_{5}\left(\phi_{0}\right) D_{\mu} \phi_{0} D^{\mu} \phi_{0}
$$

where all the functions $V_{i}$ are even and real functions of $\phi_{0}$.

\subsection{Quenched CHPT}

The modification needed to construct the quenched version of the CHPT Lagrangian in Eq. (2.3) for a generic number of flavours $N$ consists of the extension of the chiral symmetry group $S U(N)_{L} \otimes S U(N)_{R} \otimes U(1)_{V}$ to the graded group $\left[S U(N \mid N)_{L} \otimes S U(N \mid N)_{R}\right] \odot U(1)_{V}$, which enlarges the spectrum of the theory to include ghost states (the $\odot$ stands for the semidirect product of $U(1)_{V}$, which does not commute with transformations that exchange particles with ghosts). In the quenched case there are $N$ physical flavours and $N$ ghost flavours. Under the graded extension all the $N \times N$ matrices representative of the original $U(N)$ group are transformed into graded $2 \times 2$ block matrices

$$
A \rightarrow\left(\begin{array}{cc}
A & B \\
C & D
\end{array}\right)
$$

whose components are in turn $N \times N$ matrices. The matrices $A$ and $D(B$ and $C$ ) have bosonic (fermionic) character. The trace is then transformed into supertrace:

$$
\operatorname{tr}(A) \rightarrow \operatorname{str}\left(\begin{array}{cc}
A & B \\
C & D
\end{array}\right)=\operatorname{tr}(A)-\operatorname{tr}(D) .
$$

The leading order Lagrangian of quenched CHPT can be written in full analogy to the standard CHPT case[?

$$
\begin{aligned}
\mathcal{L}_{2}= & V_{1}\left(\Phi_{0}\right) \operatorname{str}\left(D_{\mu} U_{s} D^{\mu} U_{s}^{\dagger}\right)+V_{2}\left(\Phi_{0}\right) \operatorname{str}\left(\chi_{s}^{\dagger} U_{s}+U_{s}^{\dagger} \chi_{s}\right)-V_{0}\left(\Phi_{0}\right) \\
& +V_{5}\left(\Phi_{0}\right) D_{\mu} \Phi_{0} D^{\mu} \Phi_{0},
\end{aligned}
$$

\footnotetext{
${ }^{3}$ To distinguish between a quenched CHPT quantity and its standard counterpart we use either capital letters (as in $\phi \rightarrow \Phi$ ) or, when this is not possible, the $s$ subscript (as in $U \rightarrow U_{s}$ ).
} 
where again $V_{i}\left(\Phi_{0}\right)$ are even and real functions of the generalized singlet field $\Phi_{0}$. The graded meson field is defined through the usual exponential representation:

$$
U_{s}=\exp (\sqrt{2} i \Phi / F)
$$

where $F$ is the bare quenched pion decay constant and $\Phi$ is now a hermitian non traceless $2 \times 2$ block matrix

$$
\Phi=\left(\begin{array}{cc}
\phi & \theta^{\dagger} \\
\theta & \tilde{\phi}
\end{array}\right), \quad \operatorname{str}(\Phi)=\Phi_{0}=\phi_{0}-\tilde{\phi}_{0},
$$

which contains the new ghost states of the quenched spectrum. All the possible quenched meson states carry the quantum numbers of a two particle bound state made up with quarks $q$ or ghost-quarks $\tilde{q}$. On the diagonal sites it contains the physical pseudo-Goldstone boson matrix $\phi$ (i.e. the physical pions including the singlet component), with the quantum numbers of a $q \bar{q}$ pair, and the ghost field matrix $\tilde{\phi}$, with the quantum numbers of a $\tilde{q} \overline{\tilde{q}}$ pair. They are both of bosonic nature. In the off-diagonal sites are the ghost hybrid fields $\theta$ and $\theta^{\dagger}$, which carry the quantum numbers of a mixed $\tilde{q} \bar{q}$ and $q \overline{\tilde{q}}$ pair respectively, both of fermionic nature. This spectrum of meson states can be found also in the original derivation by Morel [1]. He calculated the functional integral over the quark and ghostquark fields (in the leading large- $d$ expansion and strong gauge coupling limit) and obtained exactly the meson spectrum of quenched CHPT, with mesons of composite nature, given by bilinears of quarks/ghost-quarks at the same lattice site.

The covariant derivative over the field $U_{s}$ is defined as $D^{\mu} U_{s}=\partial^{\mu} U_{s}-$ $i r_{s}^{\mu} U_{s}+i U_{s} l_{s}^{\mu}$, where $r_{s}^{\mu}\left(l_{s}^{\mu}\right)$ is the right(left)-handed external source of the graded group. The field $\chi_{s}=2 B_{0}\left(s_{s}+i p_{s}\right)$ contains the external scalar $\left(s_{s}\right)$ and pseudoscalar $\left(p_{s}\right)$ sources analogously to the ordinary CHPT case. All the external fields $r_{s}^{\mu}, l_{s}^{\mu}, s_{s}, p_{s}$ are generalizations of the standard external fields, in order to make the Lagrangian in Eq. (2.4) locally invariant under the graded group $\left[S U_{L}(N \mid N) \otimes S U_{R}(N \mid N)\right] \odot U(1)_{V}$. Since we are not interested in studying matrix elements containing the spurious fields as external legs, we will always use the standard external sources only. With this reduction a generic graded source reads as follows:

$$
j_{s}=\left(\begin{array}{ll}
j & 0 \\
0 & 0
\end{array}\right), j=p, v_{\mu}, a_{\mu} .
$$

For the scalar external source we must recall that it is defined to contain the quark mass matrix $\mathcal{M}$ which has to be the same both for the quarks and the ghosts:

$$
s_{s}=\left(\begin{array}{cc}
\mathcal{M}+\delta s & 0 \\
0 & \mathcal{M}
\end{array}\right) .
$$

In what follows the quark mass matrix will be taken proportional to the unit matrix: $\mathcal{M}=m_{q} \mathbf{1}$. All the Goldstone bosons and their ghost counterparts will 
have the same mass: $M^{2}=2 B_{0} m_{q}$. We have adopted the usual CHPT notation and call $M^{2}$ the lowest order term in the expansion of the mass of the pions in powers of quark masses:

$$
M_{\pi}^{2}=2 B_{0} m_{q}+O\left(m_{q}^{2}\right)=M^{2}+O\left(m_{q}^{2}\right) .
$$

Finally, we expand the functions $V_{i}\left(\Phi_{0}\right)$ in powers of $\Phi_{0}$ :

$$
\begin{aligned}
V_{0}\left(\Phi_{0}\right) & =\frac{m_{0}^{2}}{2 N_{c}} \Phi_{0}^{2}+O\left(\Phi_{0}^{4}\right), \\
V_{1,2}\left(\Phi_{0}\right) & =\frac{F^{2}}{4}+\frac{1}{2} v_{1,2} \Phi_{0}^{2}+O\left(\Phi_{0}^{4}\right), \\
V_{5}\left(\Phi_{0}\right) & =\frac{\alpha}{2 N_{c}}+O\left(\Phi_{0}^{2}\right),
\end{aligned}
$$

and we shall always work with number of colours $N_{c}=3$.

\section{One-loop divergences}

To calculate the ultraviolet divergent part of the quenched generating functional to one loop we use the background field method, i.e. expand the action around the classical solution, which is determined by the external sources through the classical equations of motion. We write the field $U_{s}$ as:

$$
U_{s}=u_{s} e^{i \Xi} u_{s}
$$

where $\bar{U}_{s}=u_{s}^{2}$ is the classical solution to the equations of motion. In the absence of spurious external sources it reduces to

$$
u_{s}=\left(\begin{array}{ll}
u & 0 \\
0 & \mathbf{1}
\end{array}\right) \text {. }
$$

We decompose the fluctuation $\Xi$ similarly to the field $\Phi$ and write:

$$
\Xi=\left(\begin{array}{cc}
\xi & \zeta^{\dagger} \\
\zeta & \tilde{\xi}
\end{array}\right), \quad \operatorname{str}(\Xi)=\sqrt{N}\left(\xi_{0}-\tilde{\xi}_{0}\right),
$$

(note that with this normalization the $\xi_{0}$ and $\tilde{\xi}_{0}$ have a proper kinetic term). The matrix fields $\xi$ and $\zeta$ are decomposed as follows

$$
\xi=\sum_{a=0}^{N^{2}-1} \hat{\lambda}_{a} \xi^{a}, \quad \zeta=\sum_{a=0}^{N^{2}-1} \hat{\lambda}_{a} \zeta^{a},
$$

and the fields $\tilde{\xi}$ and $\zeta^{\dagger}$ analogously, where $\hat{\lambda}_{a}=\lambda_{a} / \sqrt{2}, a=1, \ldots, N^{2}-1$, and $\hat{\lambda}_{0}=1 / \sqrt{N}$. Given their special character, it is useful to separate the singlet components of the $\xi$ and $\tilde{\xi}$ fields from the rest, and combine them into one vector:

$$
X_{0}=\left(\begin{array}{c}
\xi_{0} \\
\tilde{\xi}_{0}
\end{array}\right) \text {. }
$$


The remaining fields are put into components of the following vectors:

$$
\xi^{T}=\left(\xi^{1}, \xi^{2}, \ldots, \xi^{N^{2}-1}\right), \quad \zeta^{\dagger}=\left(\zeta^{\dagger 0}, \zeta^{\dagger 1}, \ldots, \zeta^{\dagger N^{2}-1}\right)
$$

With this notation the action can be written as

$$
\begin{aligned}
S[\Phi] & =S[\bar{\Phi}]-\frac{F^{2}}{4} \int d x\left\{X_{0}^{T} D_{X} X_{0}+\xi^{T} D_{\xi} \xi+\xi_{0} B^{T} \xi+\xi^{T} B \xi_{0}+2 \zeta^{\dagger} D_{\zeta} \zeta\right. \\
& \left.-\tilde{\xi}^{T}\left(\square+M^{2}\right) \tilde{\xi}\right\}+O\left(\Xi^{3}\right) .
\end{aligned}
$$

The explicit expressions for the various differential operators $D_{X, \xi, \zeta}$ and the matrix $B$ will be given below. The matrix $B$ induces a mixing between the singlet and non singlet component of the physical meson field. Notice also that the fields $\tilde{\xi}$ are completely decoupled from the rest: the integration over these degrees of freedom produces only an irrelevant constant.

Before deriving the various contributions to the generating functional at one loop we shift the field $\xi$ in order to remove the mixing with the singlet component $\xi_{0}$. By performing the translation

$$
\xi=\xi^{\prime}-D_{\xi}^{-1} B \xi_{0}
$$

one gets

$$
\xi^{T} D_{\xi} \xi+\xi_{0} B^{T} \xi+\xi^{T} B \xi_{0}=\xi^{T} D_{\xi} \xi^{\prime}-\xi_{0} B^{T} D_{\xi}^{-1} B \xi_{0}
$$

In this manner the action up to the quadratic fluctuations becomes a sum of quadratic differential forms diagonal in the fields $X_{0}, \xi, \tilde{\xi}, \zeta, \zeta^{\dagger}$. The price to pay is that now the differential operator acting on the singlet field $X_{0}$ has a nonlocal term. Denoting as $\bar{D}_{X}$ the new non local operator acting on $X_{0}$ after the shift, the quenched generating functional to one loop can be formally written as follows

$$
e^{i Z_{1 \text { loop }}^{\mathrm{qCHPT}}}=\mathcal{N} \frac{\operatorname{det} D_{\zeta}}{\left(\operatorname{det} D_{\xi}\right)^{\frac{1}{2}}\left(\operatorname{det} \bar{D}_{X}\right)^{\frac{1}{2}}} .
$$

As we will see below, the non locality of $\bar{D}_{X}$ will hardly make the calculation of the divergent part more complicated.

\subsection{Integral over the $\xi$ fields}

The differential operator $D_{\xi}^{a b}$ is defined as follows

$$
\begin{aligned}
D_{\xi}^{a b} \xi_{b} & =d_{\mu} d^{\mu} \xi^{a}+\hat{\sigma}^{a b} \xi_{b}, \\
d_{\mu} \xi^{a} & =\partial_{\mu} \xi^{a}+\hat{\Gamma}_{\mu}^{a b} \xi_{b},
\end{aligned}
$$

\footnotetext{
${ }^{4}$ We remind the reader that the indices $a, b$ run from 1 to $N^{2}-1$. The singlet components are treated separately.
} 
where

$$
\hat{\Gamma}_{\mu}^{a b}=-\left\langle\Gamma_{\mu}\left[\hat{\lambda}^{a}, \hat{\lambda}^{b}\right]\right\rangle, \quad \hat{\sigma}^{a b}=-\frac{1}{4}\left\langle\left[u_{\mu}, \hat{\lambda}^{a}\right]\left[u^{\mu}, \hat{\lambda}^{b}\right]\right\rangle+\frac{1}{4}\left\langle\left\{\hat{\lambda}^{a}, \hat{\lambda}^{b}\right\} \chi_{+}\right\rangle,
$$

and $\Gamma_{\mu}=1 / 2\left(\left[u^{\dagger}, \partial_{\mu} u\right]-i u^{\dagger} r_{\mu} u-i u l_{\mu} u^{\dagger}\right)$ is the vector current connection of the covariant derivative over the dynamical fields.

The ultraviolet divergent part of the integral over the $\xi$ fields can be derived in closed form by regularizing the determinant in $d$ dimensions and using standard heat-kernel techniques. The result reads:

$$
\begin{aligned}
\frac{i}{2} \ln \operatorname{det} D_{\xi} & =-\frac{1}{(4 \pi)^{2}(d-4)} \int d x\left\{\frac{N}{6}\left\langle\Gamma_{\mu \nu} \Gamma^{\mu \nu}\right\rangle+\frac{1}{2}\left[\frac{1}{4}\left\langle u_{\mu} u_{\nu}\right\rangle\left\langle u^{\mu} u^{\nu}\right\rangle+\frac{1}{8}\left\langle u_{\mu} u^{\mu}\right\rangle^{2}\right.\right. \\
& +\frac{N}{8}\left\langle\left(u_{\mu} u^{\mu}\right)^{2}\right\rangle+\frac{N}{4}\left\langle u_{\mu} u^{\mu} \chi_{+}\right\rangle+\frac{1}{4}\left\langle u_{\mu} u^{\mu}\right\rangle\left\langle\chi_{+}\right\rangle \\
& +\left(\frac{N}{8}-\frac{1}{2 N}\right)\left\langle\chi_{+}^{2}\right\rangle+\left(\frac{1}{8}+\frac{1}{4 N^{2}}\right)\left\langle\chi_{+}\right\rangle^{2} \\
& \left.\left.-\frac{1}{2}\left\langle u_{\mu}\right\rangle\left\langle u^{\mu}\left(u_{\nu} u^{\nu}+\chi_{+}\right)\right\rangle\right]\right\}+\ldots
\end{aligned}
$$

where the ellipsis stands for contributions which are finite in four dimensions. This result is the standard CHPT result derived in [9], where now we also keep terms proportional to $\left\langle u_{\mu}\right\rangle$ that are nonzero only in the presence of the singlet component.

\subsection{Integral over the $\zeta$ fields}

The differential operator $D_{\zeta}^{a b}$ is defined like in Eq. (3.5), but with barred quantities, given by

$$
\bar{\Gamma}_{\mu}^{a b}=-\left\langle\Gamma_{\mu} \hat{\lambda}^{a} \hat{\lambda}^{b}\right\rangle, \quad \bar{\sigma}^{a b}=\frac{1}{4}\left\langle\left(u_{\mu} u^{\mu}+\chi_{+}+4 B_{0} \mathcal{M}\right) \hat{\lambda}^{a} \hat{\lambda}^{b}\right\rangle,
$$

where we recall that $\mathcal{M}$ is the quark mass matrix.

The ultraviolet divergent part of the functional integral over the $\zeta$ fields can also be given in closed form using standard heat-kernel techniques. The result reads:

$i \ln \operatorname{det} D_{\zeta}=\frac{-1}{(4 \pi)^{2}(d-4)} \int d x\left[\frac{N}{6}\left\langle\Gamma_{\mu \nu} \Gamma^{\mu \nu}\right\rangle+\frac{N}{16}\left\langle\left(u_{\mu} u^{\mu}+\chi_{+}+4 B_{0} \mathcal{M}\right)^{2}\right\rangle\right]+\ldots$.

As we remarked in Ref. [10], the integral over the $\zeta$ fields completely removes the terms linear in $N$ in the divergences of standard CHPT to one loop. This

\footnotetext{
${ }^{5}$ Here the singlet component is included, and the indices $a, b$ run from 0 to $N^{2}-1$.
} 
dependence is not fully explicit in Eq. (3.7), since a factor $N$ is contained in the trace of $\chi_{+}$, when we expand this around $s=\mathcal{M}$ and for $\mathcal{M}$ diagonal:

$$
\left\langle\chi_{+}\right\rangle=2 N M^{2}+O\left(\phi^{2}\right)
$$

This result shows that the qCHPT scheme is coherent: the terms linear in $N$ can only be generated by quark loops, and these are supposed to be absent in the quenched approximation.

\subsection{Integral over the $X_{0}$ fields}

After the shift of the $\xi$ field the operator acting on $X_{0}$ can be written as:

$$
X_{0}^{T} \bar{D}_{X} X_{0}=X_{0}^{T}\left[D_{X}-\frac{1}{2}\left(1+\tau_{3}\right) B^{T} D_{\xi}^{-1} B\right] X_{0}
$$

where

$$
\begin{aligned}
D_{X} & =D_{X}^{0}+A_{X} \\
D_{X}^{0} & =\tau_{3}\left(\square+M^{2}\right)+\frac{N}{3}\left(1-\tau_{1}\right)\left(\alpha \square+m_{0}^{2}\right) \\
A_{X} & =\frac{1}{4 N}\left(1+\tau_{3}\right)\left\langle\hat{\chi}_{+}\right\rangle-N\left(1-\tau_{1}\right)\left(v_{1}\left\langle u_{\mu} u^{\mu}\right\rangle+v_{2}\left\langle\hat{\chi}_{+}\right\rangle\right)+O\left(\Phi_{0}^{2}\right) \\
B^{a} & =\frac{1}{2 \sqrt{2 N}}\left\langle\lambda^{a} \chi_{+}\right\rangle
\end{aligned}
$$

and $\hat{\chi}_{+}=\chi_{+}-2 M^{2} \mathbf{1}$, so that $\left\langle\hat{\chi}_{+}\right\rangle=\left\langle\chi_{+}\right\rangle-2 N M^{2}$. The expression of $D_{X}^{0}$, the "free" part of the differential operator, clearly shows that the theory has a problem here: it is not possible to diagonalize that operator, and we do not have two freely propagating normal fields $\left(\xi_{0}, \tilde{\xi}_{0}\right)$. On the other hand this problem is welcome in this context, since it is thought to be the manifestation of the absence of quark loops in the singlet field propagator, at the level of the effective theory. In the language of Feynman diagrams this problem shows up as a double pole in the propagator of the singlet field, whose consequences on observables have been studied by several authors [2, 3, 12]. We adopt the usual point of view on this problem, i.e. assume that it has to be there, and proceed with the calculation of the divergent part of the generating functional.

In this case we cannot apply straightforwardly the heat-kernel techniques, because the differential operator does not reduce to a diagonal Klein-Gordon operator when the external fields are put to zero. Therefore we just expand the logarithm of the differential operator, and isolate the ultraviolet divergent terms:

$$
\begin{aligned}
\operatorname{Tr} \ln \left(\bar{D}_{X} / D_{X}^{0}\right) & =\operatorname{Tr}\left[D_{X}^{0-1}\left(\bar{D}_{X}-D_{X}^{0}\right)\right] \\
& -\frac{1}{2} \operatorname{Tr}\left[D_{X}^{0-1}\left(\bar{D}_{X}-D_{X}^{0}\right) D_{X}^{0}{ }^{-1}\left(\bar{D}_{X}-D_{X}^{0}\right)\right]+\ldots
\end{aligned}
$$


One can easily see that the ellipsis in (3.12) contains ultraviolet finite terms only. We postpone a more detailed discussion of the infrared behaviour of Eq. (3.12) to the end of this section. The inverse of the "free" operator $D_{X}^{0}$ is:

$$
D_{X}^{0-1}=G_{0}\left[\tau_{3}-\left(1+\tau_{1}\right) \frac{N}{3}\left(\alpha \square+m_{0}^{2}\right) G_{0}\right],
$$

where

$$
\left(\square+M^{2}\right)_{x} G_{0}(x-y)=\delta(x-y)
$$

and

$$
\bar{D}_{X}-D_{X}^{0}=A_{X}-\frac{1}{2}\left(1+\tau_{3}\right) B^{T} D_{\xi}^{-1} B .
$$

As we anticipated above, the overall effect of the shift made to remove the mixing between singlet and nonsinglet fields is easily accounted for. Expanding around the free part of $D_{\xi}^{-1}$ in the non local term one gets

$$
B^{T} D_{\xi}^{-1} B=\frac{1}{4 N} G_{0}\left[\left\langle\chi_{+}^{2}\right\rangle-\frac{1}{N}\left\langle\chi_{+}\right\rangle^{2}\right]+O\left(G_{0}^{2}\right) .
$$

The term proportional to $O\left(G_{0}^{2}\right)$ can only yield ultraviolet finite contributions to Eq. (3.12), while the $G_{0}$ term yields ultraviolet divergent contributions only to the first term of the expansion in Eq. (3.12). This shows that also in the singlet sector the UV divergent part is local and can be given in closed form. The calculation of the ultraviolet divergent part of $\ln \operatorname{det} \bar{D}_{X}$ is now easy: we simply have to insert back Eqs. (3.13, 3.15) into Eq. (3.12) and keep only the UV divergent parts. Having worked out the traces (over the $\tau$ matrices) we obtain:

$$
\begin{aligned}
\frac{i}{2} \operatorname{Tr} \ln \left(\bar{D}_{X} / D_{X}^{0}\right)= & -\frac{1}{(4 \pi)^{2}(d-4)}\left\{\frac{1}{4 N}\left\langle\chi_{+}^{2}\right\rangle-\frac{1}{8 N^{2}}\left\langle\chi_{+}\right\rangle^{2}\right. \\
& +\frac{m_{0}^{2}}{6}\left\langle\chi_{+}\right\rangle-\frac{\alpha}{12}\left\langle\chi_{+}^{2}\right\rangle+\frac{\alpha^{2}}{72}\left\langle\hat{\chi}_{+}\right\rangle^{2} \\
& \left.-\frac{1}{2}\left\langle\hat{\chi}_{+}\right\rangle\left(v_{1}\left\langle u_{\mu} u^{\mu}\right\rangle+v_{2}\left\langle\hat{\chi}_{+}\right\rangle\right)\right\}+\ldots,
\end{aligned}
$$

where the ellipsis contains UV finite terms only. The terms proportional to inverse powers of $N$ exactly cancel those contained in Eq. (3.7) giving a result that is totally $N$-independent. The terms proportional to $m_{0}^{2}$ and powers of $\alpha$ are the effect of the double pole in the singlet propagator, and are also $N$-independent. Note that no mixed terms of the type $\left(m_{0}^{2}, \alpha\right) \times\left(v_{1}, v_{2}\right)$ can be produced in the divergent part.

The term proportional to $m_{0}^{2}$ is a term already present in the $O\left(p^{2}\right)$ Lagrangian. To remove that divergence one has to add to the lowest order parameter $B_{0}$ in the $\mathcal{L}_{2}$ Lagrangian a $d$-dependent part proportional to $m_{0}^{2}$ that has a pole at $d=4$ :

$$
B_{0} \rightarrow B_{0}\left[1+\frac{\mu^{d-4}}{16 \pi^{2}} \frac{1}{d-4} \frac{2 m_{0}^{2}}{3 F^{2}}+b_{0}(\mu)\right]
$$


This feature is completely new with respect to standard CHPT (in dimensional regularization), and stems from the fact that in the quenched theory we have a new mass scale that does not vanish in the chiral limit. After the divergence has been removed, we are left with a term of the form $m_{0}^{2} \ln M^{2}\left\langle\chi_{+}\right\rangle$. This term contains all the one-loop quenched chiral logs that have been discussed at length in the literature. Our calculation shows that they can be fully accounted for by defining a renormalized constant $\bar{B}_{0}$

$$
B_{0} \rightarrow \bar{B}_{0}=B_{0}\left(1-\frac{m_{0}^{2}}{48 \pi^{2} F^{2}} \ln \frac{M^{2}}{\mu^{2}}+b_{0}(\mu)\right)
$$

Notice that since $B_{0}$ is independent from the quark masses, $\bar{B}_{0}$ becomes divergent in the chiral limit. To find evidence for these quenched chiral logs one should try to extract from lattice data this quantity $\bar{B}_{0}$. As we will see the quark condensate and the scalar form factor are two excellent candidates for this, since they are the simplest quantities which are explicitly proportional to $\bar{B}_{0}$. Other quantities will tipically depend on $\bar{B}_{0}$ through the renormalized pion mass. This at one loop is given by:

$$
M_{\pi}^{2}=2 \bar{B}_{0} m_{q}+O\left(m_{q}^{2}\right)
$$

and is not divergent in the chiral limit. These other quantities are therefore much less suitable to identify the presence of quenched chiral logs.

Of course what we have just said is valid in the specific sector we are studying here. To extend it to other sectors of the effective theory (like the non-leptonic weak interactions) requires further study. However we have a rather simple argument that shows that what has happened here will happen also in other sectors: the quenched chiral logs to one loop contribute to the redefinition of one of the constants appearing in the lowest order Lagrangian. In order not to interrupt the discussion here we relegate the argument to Appendix A.

\subsection{Complete result}

In this section we put together all the various pieces and give the complete result for the ultraviolet divergent part of the generating functional of qCHPT to one loop. The explicit expression for Eq. (3.4) is:

$$
\begin{aligned}
Z_{1 \text { loop }}^{\mathrm{qCHPT}}= & -\frac{1}{(4 \pi)^{2}(d-4)} \int d x\left[\frac{1}{8}\left\langle u_{\mu} u_{\nu}\right\rangle\left\langle u^{\mu} u^{\nu}\right\rangle+\frac{1}{16}\left\langle u_{\mu} u^{\mu}\right\rangle^{2}\right. \\
& +\frac{1}{8}\left(1-4 v_{1}\right)\left\langle u_{\mu} u^{\mu}\right\rangle\left\langle\hat{\chi}_{+}\right\rangle+\frac{1}{16}\left(1-8 v_{2}\right)\left\langle\hat{\chi}_{+}\right\rangle^{2} \\
& +\frac{m_{0}^{2}}{6}\left\langle\chi_{+}\right\rangle+\frac{\alpha^{2}}{72}\left\langle\hat{\chi}_{+}\right\rangle^{2}-\frac{\alpha}{12}\left\langle\chi_{+}^{2}\right\rangle \\
& \left.-\frac{1}{4}\left\langle u_{\mu}\right\rangle\left\langle u^{\mu}\left(u_{\nu} u^{\nu}+\chi_{+}\right)\right\rangle\right]+\ldots .
\end{aligned}
$$


The most striking feature of Eq. (3.21) is the complete flavour independence of the result. If we analyze in detail the modifications that the quenched approximation has produced to the divergent structure of the effective theory at the one-loop level, we come to the following list:

1. all the terms proportional to $N$ have been dropped;

2. all the terms proportional to $1 / N$ and $1 / N^{2}$ have been dropped;

3. new divergences proportional to the parameters present in the anomalous singlet sector have been produced.

All these new parameters are dimensionless, with the only exception of $m_{0}$. The dimensionless parameters ( $\alpha$ and $v_{1,2}$ ) generate divergences that have the structure of a chiral invariant term (since they do not break the chiral symmetry) of order $p^{4}$, for obvious dimensional reasons. For the same reasons $m_{0}^{2}$ generates divergences with the structure of a chiral invariant of order $p^{2}$. As it is shown in Appendix A one can very easily understand why it is only the mass term $\left\langle\chi_{+}\right\rangle$ that is generated.

As it turns out, the modifications listed in points 1. to 3. above find a very simple explanation: dropping the terms proportional to $N$ corresponds to dropping virtual quark loops. Dropping the terms proportional to $1 / N$ and $1 / N^{2}$, is a consequence of having a singlet degenerate in mass with the nonsinglet pseudoscalars. The new parameters in the singlet sector are required by the $U(1)_{A}$ anomaly, and the diseases in that sector are generated by the quenched approximation, as it is well known. These simple conclusions suggest that one could have guessed all these modifications without doing any calculation. In fact, we provide an example of how one could try such a guess in Appendix B, where we apply the same criteria to the generating functional of the non-leptonic weak interaction sector for the octet on-shell case (the complete analysis will be given elsewhere [13]), by going through the three steps we have enumerated above.

\subsection{Chiral and threshold divergences}

Quenched chiral logs are not the only problem generated by the presence of the double pole in the quenched version of the singlet propagator. As we will see in detail in Sec. 5 through several examples, this double pole generates also other kind of divergences inside contributions which are ultraviolet finite. These divergences are of two types: powerlike chiral divergences, i.e. inverse powers of $M_{\pi}^{2}$, and unphysical threshold divergences. We find it instructive, before closing this section to identify which are the terms in the generating functional which are responsible for them.

Some of the terms (and in fact an infinite series of them) that we have neglected in Eq. (3.17) because they are ultraviolet finite, contain these kind of 
singularities. They can be given in closed form only if one stops at a given order in the expansion in powers of the field $\Phi$. Since in the following sections we are not going to analyze anything beyond the four-point function, we can stop at order $\Phi^{4}$, and identify explicitly the troublesome terms. They all come from the insertion of the double pole term of (3.13) in the expansion (3.12), and give the following contribution to the generating functional:

$$
\begin{aligned}
\delta Z_{1 \text { loop }}^{\mathrm{qCHPT}} & =\frac{\left(m_{0}^{2}-\alpha M^{2}\right)}{24} \int d x d y \tilde{I}_{1}(x-y)\left\langle\hat{\chi}_{+}(y) \hat{\chi}_{+}(x)\right\rangle \\
& -\alpha \frac{\left(m_{0}^{2}-\alpha M^{2}\right)}{72} \int d x d y \tilde{I}_{1}(x-y)\left\langle\hat{\chi}_{+}(y)\right\rangle\left\langle\hat{\chi}_{+}(x)\right\rangle \\
& -\frac{\left(m_{0}^{2}-\alpha M^{2}\right)^{2}}{144} \int d x d y \tilde{I}_{2}(x-y)\left\langle\hat{\chi}_{+}(y)\right\rangle\left\langle\hat{\chi}_{+}(x)\right\rangle+O\left(\Phi^{6}\right)
\end{aligned}
$$

The functions $\tilde{I}_{1}(z), \tilde{I}_{2}(z)$ are defined in appendix Q. At infinite volume and in Minkowski space-time their Fourier transforms $I_{1}\left(q^{2}\right), I_{2}\left(q^{2}\right)$ develop an imaginary part when $q^{2} \geq 4 M_{\pi}^{2}$ which diverges at $q^{2}=4 M_{\pi}^{2}$ (see appendix Q). Moreover their values at $q^{2}=0$ are inversely proportional to $M_{\pi}^{2}$ (again see appendix C): this is the origin of powerlike chiral divergences that we will find in several observables in Sect. 5. The threshold singularities in particular make the theory meaningless in Minkowski space-time at infinite volume. In finite volume and in Euclidean space-time the same one loop functions $I_{1,2}(q)$ have been evaluated at $q^{2}=4 M_{\pi}^{2}$ in Ref. [12], and it was found that these functions give rise to enhanced finite volume corrections which are forbidden in a healthy Hamiltonian theory. As it was pointed out in Ref. [12 this shows that qCHPT can only make sense in Euclidean space-time and in finite volume.

\section{Lagrangian at order $p^{4}$}

To complete the renormalization of the quenched theory at order $p^{4}$ one needs to add the most general chiral invariant Lagrangian at this order. As in the standard CHPT case, some of the couplings appearing in the order $p^{4}$ Lagrangian have an UV divergent part in such a way that all the one-loop divergences are removed. The most general chiral invariant Lagrangian at order $p^{4}$ in standard CHPT has been given by Gasser and Leutwyler [9]. The extension to the graded symmetry version is not needed here, since we are not going beyond order $p^{4}$, and are not interested in having the spurious degrees of freedom as external particlest: we can use the standard CHPT Lagrangian right away.

There is however a slight modification that we have to introduce. As we noted before, the trace of $\chi_{+}$starts with a constant term proportional to $N$ in

\footnotetext{
${ }^{6}$ Moreover we will not consider singlet fields as external particles. They require at least two more counterterms as shown by Eq. (3.21).
} 
the degenerate mass case we are considering here. In the quenched version a linear dependence upon $N$ is forbidden, and therefore we must always substitute $\left\langle\chi_{+}\right\rangle \rightarrow\left\langle\hat{\chi}_{+}\right\rangle$. Apart from this modification, we have followed existing notations for the choice of the $O\left(p^{4}\right)$ Lagrangian, both in the $S U(3)$ and $S U(2)$ case. The $S U(3)$ choice is the standard Gasser and Leutwyler Lagrangian [9], while for $S U(2)$ we choose to use the Gasser-Sainio-Švarc Lagrangian 14.

An important point concerns the value of the counterterms: we observe that in the quenched case the counterterms do not depend on the number of flavours. Not only the divergent part, as we have explicitly shown in the previous section, but also the numerical value of the finite part of the counterterms does not change for different values of $N$. Therefore it is useful to identify, and give names to them in the general $N$ case. For the more interesting cases of $N=3$ and $N=2$, because of trace relations, one will be able to access only certain combinations of them, as we will specify below. For general $N$ the lagrangian at order $p^{4}$ is given by:

$$
\mathcal{L}_{4}=\sum_{i=0}^{10} \Lambda_{i}^{q} P_{i},
$$

where the eleven operators $P_{i}$ are listed in Table 1 (we remind the reader that in the quenched case it is necessary to change $\left.\left\langle\chi_{+}\right\rangle \rightarrow\left\langle\hat{\chi}_{+}\right\rangle\right)$. These eleven chiral invariant operators contain, besides those defined in Eq. (2.2), the following new building blocks:

$$
\begin{aligned}
f_{ \pm \mu \nu} & =u l_{\mu \nu} u^{\dagger} \pm u^{\dagger} r_{\mu \nu} u \\
\chi_{-} & =u^{\dagger} \chi u^{\dagger}-u \chi^{\dagger} u .
\end{aligned}
$$

To derive the results shown in Table 1 the following relation is useful:

$$
f_{+\mu \nu}=2 i \Gamma_{\mu \nu}-\frac{i}{2}\left[u_{\mu}, u_{\nu}\right]
$$

and the identification $\left\langle\chi_{+}^{2}\right\rangle=1 / 2\left\langle\chi_{+}^{2}+\chi_{-}^{2}\right\rangle$ and $\left\langle f_{+}^{2}\right\rangle=1 / 2\left\langle f_{+}^{2}-f_{-}^{2}\right\rangle$ can be done up to contact terms which contain external sources only.

\section{1 $S U(3)$ Lagrangian at order $p^{4}$}

For $N=3$ the Lagrangian at order $p^{4}$ reads as follows:

$$
\mathcal{L}_{4}^{(N=3)}=\sum_{i=1}^{10} L_{i}^{q} P_{i},
$$

where the operators $P_{i}$ are defined in Table 1. The $P_{0}$ operator is linearly dependent from the others through the following trace relation:

$$
P_{0}=\frac{1}{2} P_{1}+P_{2}-2 P_{3}
$$




\begin{tabular}{|c|c|c|c|c|c|}
\hline$i$ & $\begin{array}{c}P_{i} \\
\text { for } S U(N)\end{array}$ & \multicolumn{2}{|c|}{ Coeff. of $-\frac{1}{(4 \pi)^{2}(d-4)}$} & $S U(3)$ & $S U(2)$ \\
\hline 0 & $\left\langle u_{\mu} u_{\nu} u^{\mu} u^{\nu}\right\rangle$ & $\frac{N}{48}$ & 0 & Eq. (4.5) & Eq. (4.11) \\
1 & $\left\langle u_{\mu} u^{\mu}\right\rangle^{2}$ & $\frac{1}{16}$ & $\frac{1}{16}$ & $L_{1}$ & $\frac{1}{4} l_{1}$ \\
2 & $\left\langle u_{\mu} u_{\nu}\right\rangle\left\langle u^{\mu} u^{\nu}\right\rangle$ & $\frac{1}{8}$ & $\frac{1}{8}$ & $L_{2}$ & $\frac{1}{4} l_{2}$ \\
3 & $\left\langle u_{\mu} u^{\mu} u_{\nu} u^{\nu}\right\rangle$ & $\frac{N}{24}$ & 0 & $L_{3}$ & Eq. (4.11) \\
4 & $\left\langle u_{\mu} u^{\mu}\right\rangle\left\langle\chi_{+}\right\rangle$ & $\frac{1}{8}$ & $\frac{1}{8}-\frac{v_{1}}{2}$ & $L_{4}$ & $\frac{1}{8} l_{4}$ \\
5 & $\left\langle u_{\mu} u^{\mu} \chi_{+}\right\rangle$ & $\frac{N}{8}$ & 0 & $L_{5}$ & Eq. (4.11) \\
6 & $\left\langle\chi_{+}\right\rangle^{2}$ & $\frac{1}{16}+\frac{1}{8 N^{2}}$ & $\frac{1}{16}-\frac{v_{2}}{2}+\frac{\alpha^{2}}{72}$ & $L_{6}$ & $\frac{1}{16} l_{3}$ \\
7 & $\left\langle\chi_{-}\right\rangle^{2}$ & 0 & 0 & $L_{7}$ & $-\frac{1}{16} l_{7}$ \\
\hline 8 & $\frac{1}{2}\left\langle\chi_{+}^{2}+\chi_{-}^{2}\right\rangle$ & $\frac{N}{16}-\frac{1}{4 N}$ & $-\frac{\alpha}{12}$ & $L_{8}$ & Eq. (4.11) \\
9 & $-i\left\langle f_{+}^{\mu \nu} u_{\mu} u_{\nu}\right\rangle$ & $\frac{N}{12}$ & 0 & $L_{9}$ & $-\frac{1}{2} l_{6}$ \\
10 & $\frac{1}{4}\left\langle f_{+}^{2}-f_{-}^{2}\right\rangle$ & $-\frac{N}{12}$ & 0 & $L_{10}$ & $l_{5}$ \\
\hline
\end{tabular}

Table 1: List of terms of order $p^{4}$ for $N$ generic, $N=3$ and $N=2$. In the second and third columns we give the coefficient of the divergence coming from the one loop in the standard and quenched CHPT case. As we have indicated in the table, the invariants containing $\left\langle\chi_{+}\right\rangle$have to be changed with $\left\langle\chi_{+}\right\rangle \rightarrow\left\langle\hat{\chi}_{+}\right\rangle$ in the quenched case. 
which implies:

$$
L_{1}^{q}=\Lambda_{1}^{q}+\frac{1}{2} \Lambda_{0}^{q}, \quad L_{2}^{q}=\Lambda_{2}^{q}+\Lambda_{0}^{q}, \quad L_{3}^{q}=\Lambda_{3}^{q}-2 \Lambda_{0}^{q},
$$

In order to reabsorb the divergences at one loop we define the $L_{i}^{q}$ in the following manner:

$$
\begin{aligned}
L_{i}^{q} & =L_{i}^{q r}(\mu)+\Gamma_{i}^{q} \lambda, \\
\lambda & =\frac{\mu^{d-4}}{16 \pi^{2}}\left[\frac{1}{d-4}-\frac{1}{2}\left(\ln 4 \pi+\Gamma^{\prime}(1)+1\right)\right],
\end{aligned}
$$

with $\mu$ the renormalization scale, $\lambda$ contains the divergence at $d=4$ and the coefficients $\Gamma_{i}^{q}$ are given by

$$
\begin{array}{rlrl}
\Gamma_{1}^{q}=\frac{1}{16}, & \Gamma_{2}^{q}=\frac{1}{8}, & \Gamma_{4}^{q}=\frac{1}{8}\left(1-4 v_{1}\right), \\
\Gamma_{6}^{q}=\frac{1}{16}\left(1-8 v_{2}+\frac{2}{9} \alpha^{2}\right), & \Gamma_{8}=-\frac{\alpha}{12},
\end{array}
$$

all the other $\Gamma_{i}^{q}$ are zero.

\section{2 $S U(2)$ Lagrangian at order $p^{4}$}

For $N=2$ the Lagrangian at order $p^{4}$ reads as follows:

$$
\mathcal{L}_{4}^{(N=2)}=\sum_{i=1}^{7} l_{i}^{q} Q_{i}
$$

where

$$
\begin{aligned}
Q_{1} & =\frac{1}{4}\left\langle u_{\mu} u^{\mu}\right\rangle^{2}, \\
Q_{2} & =\frac{1}{4}\left\langle u_{\mu} u_{\nu}\right\rangle\left\langle u^{\mu} u^{\nu}\right\rangle, \\
Q_{3} & =\frac{1}{16}\left\langle\hat{\chi}_{+}\right\rangle^{2}, \\
Q_{4} & =\frac{1}{8}\left\langle u_{\mu} u^{\mu}\right\rangle\left\langle\hat{\chi}_{+}\right\rangle, \\
Q_{5} & =\frac{1}{4}\left\langle f_{+}^{2}-f_{-}^{2}\right\rangle, \\
Q_{6} & =\frac{i}{2}\left\langle f_{+}^{\mu \nu} u_{\mu} u_{\nu}\right\rangle, \\
Q_{7} & =-\frac{1}{16}\left\langle\chi_{-}\right\rangle^{2} .
\end{aligned}
$$


To reduce the number of chiral invariants needed we have used the following relations:

$$
P_{0}=-\frac{1}{2} P_{1}+P_{2}, \quad P_{3}=\frac{1}{2} P_{1}, \quad P_{5}=\frac{1}{2} P_{4}, \quad P_{8}=\frac{1}{2}\left(P_{6}+P_{7}\right) .
$$

which imply the following relations between the $N=3$ and $N=2$ counterterms:

$$
\begin{array}{rlrl}
\frac{1}{4} l_{1}^{q} & =L_{1}^{q}+\frac{1}{2} L_{3}^{q}, & \frac{1}{4} l_{2}^{q} & =L_{2}^{q}, \quad \frac{1}{16} l_{3}^{q}=L_{6}^{q}+\frac{1}{2} L_{8}^{q}, \\
\frac{1}{8} l_{4}^{q}=L_{4}^{q}+\frac{1}{2} L_{5}^{q}, & -\frac{1}{16} l_{7}^{q}=L_{7}^{q}+\frac{1}{2} L_{8}^{q} .
\end{array}
$$

Note that the trace relations have been written down using the invariant $\left\langle\chi_{+}\right\rangle$, and must be reexpressed in terms of $\left\langle\hat{\chi}_{+}\right\rangle$in the quenched case. This generates a correction to the constants appearing in the $\mathcal{L}_{2}$ Lagrangian, see below. In order to reabsorb the divergences at one loop we define the $l_{i}^{q}$ in the following manner:

$$
l_{i}^{q}=l_{i}^{q r}(\mu)+\gamma_{i}^{q} \lambda,
$$

with:

$$
\begin{aligned}
& \gamma_{1}^{q}=\frac{1}{4}, \quad \gamma_{2}^{q}=\frac{1}{2}, \quad \gamma_{3}^{q}=1-8 v_{2}-\frac{2}{3} \alpha+\frac{2}{9} \alpha^{2}, \\
& \gamma_{4}^{q}=1-4 v_{1}, \quad \gamma_{7}^{q}=\frac{2}{3} \alpha,
\end{aligned}
$$

all the other $\gamma_{i}^{q}$ are zero. We find useful for the analysis of the phenomenology to introduce the scale independent constants $\bar{l}_{i}^{q}$, defined as follows:

$$
\bar{l}_{i}^{q}=\frac{32 \pi^{2}}{\gamma_{i}^{q}} l_{i}^{q r}(\mu)-\ln \frac{M^{2}}{\mu^{2}} .
$$

As we mentioned above, a complete renormalization at the one-loop level requires, in the quenched case, the renormalization of the order $p^{2}$ constant $B_{0}$ due to divergences proportional to $m_{0}^{2}$. In the present case, $\left\langle\chi_{+}^{2}\right\rangle$ has been eliminated with the use of the Cayley-Hamilton relations (4.11) in favour of $\left\langle\hat{\chi}_{+}\right\rangle^{2}$ and $M^{2}\left\langle\chi_{+}\right\rangle$. The divergence proportional to the latter can also be reabsorbed in the renormalization of the $B_{0}$ parameter. Since $P_{5}=4 Q_{4}+2 M^{2}\left\langle u_{\mu} u^{\mu}\right\rangle$ the constant $F^{2}$ receives a finite correction proportional to $L_{5}^{q}$. For later convenience, we define here the renormalized constants at order $p^{2}$ in the two-flavour case, in such a way that they include also finite corrections:

$$
\begin{aligned}
\overline{\mathcal{L}}_{2}= & \frac{\bar{F}^{2}}{4}\left\langle u_{\mu} u^{\mu}+\bar{\chi}_{+}\right\rangle, \\
\bar{F}^{N=2}= & F\left(1+4 L_{5}^{q} \frac{M^{2}}{F^{2}}\right), \\
\bar{B}_{0}^{N=2}= & B_{0}\left[1-\frac{\left(m_{0}^{2}-2 \alpha M^{2}\right)}{48 \pi^{2} F^{2}}\left(\ln \frac{M^{2}}{\mu^{2}}+1\right)\right. \\
& \left.\quad-\left(8 L_{5}^{q}+\frac{\alpha}{48 \pi^{2}}\right) \frac{M^{2}}{F^{2}}+b_{0}(\mu)\right],
\end{aligned}
$$


where, with an obvious notation $\bar{\chi}_{+}$stands for the analogous of $\chi_{+}$which contains $\bar{B}_{0}$ instead of $B_{0}$.

\section{Analysis of various observables in quenched CHPT}

In this section we make a complete one-loop analysis of several observables in quenched CHPT. The main reason for this is to study the problems generated by quenching in the finite part of the one-loop corrections, which we have not considered in the generating functional. As we will see, some of the finite corrections diverge in the chiral limit. The origin of these divergences can be traced back to the presence of the double pole in the singlet two-point function. The double pole carries in the numerator a new mass scale $m_{0}$ that does not vanish in the chiral limit, and hence modifies the chiral power counting valid in CHPT. The standard power counting goes as follows: the chiral order of a generic diagram is given by the simple formula

$$
D_{g}=4 L-2 I+\sum_{d} d N_{d}
$$

where $D_{g}$ is the chiral dimension of a graph $g$ that has $L$ loops, $I$ internal lines, and $N_{d}$ vertices of chiral dimension $d$. The topological relation

$$
L=I-V+1
$$

where $V=\sum_{d} N_{d}$ is the total number of vertices, can be used to obtain

$$
D_{g}=2 L+\sum_{d}(d-2) N_{d}+2 .
$$

Since in standard CHPT the lowest chiral dimension of a vertex is two, the chiral dimension of a graph is always bigger than two, and increases with the number of loops and vertices with chiral dimension bigger than two. In quenched CHPT the situation changes, and we have to allow for the presence of vertices with chiral order zero, i.e. the insertions on the singlet propagators proportional to $m_{0}^{2}$ (that is a constant in the chiral limit). In this case Eqs. (5.1) through (5.3) are still valid, but due to the presence of terms with $d=0, D_{g}$ may now be smaller than two, and even negative. Naively one could conclude that $D_{g}$ could even be unbounded from below. However, one has to take into account the fact that virtual quark loops are forbidden: this puts a series of constraints on the type of graphs with $m_{0}^{2}$ insertions that are allowed. For example: it is not possible for two $m_{0}^{2}$ vertices to lie on the same line one after the other, or, no standard vertices can have all the outgoing lines that end up on an $m_{0}^{2}$ vertex. These

\footnotetext{
${ }^{7}$ There is one exception to this, given by vertices with physical external sources. In this case disconnected quark loops are allowed, since they are not generated by the QCD determinant (see Section 5.2).
} 
constraints are such that $D_{g}$ comes out to be bounded from below, although it may be negative. The value of the lower bound depends on the observable - we will see explicit examples below.

In what follows we are going to analyze: the quark condensate, the pion mass and decay constant, the vector and scalar form factors of the pion and the $\pi \pi$ scattering amplitude. Although these quantities (with the exception of the form factors) were already analyzed at the one-loop level in previous works [2, 20, 12], we find it useful to present them here again, in view of the renormalization that we have performed at the level of the generating functional, and also of our definition of the lagrangian at order $p^{4}$. We make the analysis in the case of two light flavours with degenerate masses.

\subsection{Quark condensate, pion mass and decay constant}

As anticipated in the previous section the renormalized scalar quark condensate plays a crucial role among the quenched observables in the strong sector, since it contains an explicit dependence upon the quenched chiral logarithms through the $\bar{B}_{0}$ parameter (4.16) (everywhere in this section we shall use the $\bar{B}_{0}$ parameter as defined in Eq. (4.16), dropping the $N=2$ superscript). We shall see later in the case of the scalar form factor that all the $\bar{q} q$ matrix elements share the same feature. The renormalized scalar density to one loop in the two-flavour case is given by

$$
\langle\bar{q} q\rangle_{q}=-F_{\pi}^{2} \bar{B}_{0}\left[1+O\left(M^{2}\right)\right],
$$

where we have not written down explicitly the standard chiral corrections of order $M^{2}$. The problem with these corrections is that they contain contributions coming from counterterms of order $p^{4}$ that contain only external fields (we have not written them down in the previous section). These counterterms cannot be determined on a phenomenological basis: their presence in the expression of the quark condensate reflects the fact that away from the chiral limit, this quantity cannot be defined unambiguously. We refer the reader to Ref. [8] for a detailed discussion of this point. On the other hand, in the chiral limit, where this ambiguity disappears, the quark condensate diverges due to the quenched chiral logarithms inside $\bar{B}_{0}$.

The pion decay constant to one loop is renormalized only by a finite amount in the quenched two-flavour case: $F_{\pi}=\bar{F}$, see Eq. (4.16). Notice that in the quenched three-flavour case there is no need to define an $\bar{F}$, but on the other hand $L_{5}^{q}$ directly contributes to $F_{\pi}$ in such a way that for $N=3$ and $N=2$ (as also for any other $N$ ) one has the same pion decay constant, as expected[? The diagrams which renormalize the pion mass to one loop are shown in Fig. 1. The meson tadpole and its ghost counterpart cancel each other, so that the

\footnotetext{
${ }^{8}$ We thank Martin Lüscher for pointing out an inconsistency on this point in the first version of the manuscript.
} 

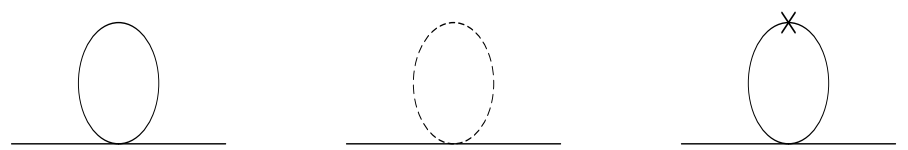

Figure 1: One-loop diagrams in quenched CHPT that contribute to the squared pion mass $M_{\pi}^{2}$. They are the meson tadpole, its ghost counterpart and the tadpole with one singlet vertex $(x)$ insertion.

renormalization of the quenched pion mass at one loop is provided by the tadpole with one singlet vertex insertion and its counterterm:

$$
M_{\pi}^{2}=2 \bar{B}_{0} m_{q},
$$

where $m_{q}$ is the light quark mass. As one can see, all the one-loop corrections, including the quenched chiral logarithm have been reabsorbed in $\bar{B}_{0}$. Since $\bar{B}_{0} m_{q} \sim m_{q} \log m_{q}$ when approaching the chiral limit, the renormalized pion mass tends to zero like $m_{q} \log m_{q}$. No divergence is produced by quenching in the behaviour of the renormalized pion mass in the chiral limit, although the way it approaches zero is different from that of standard CHPT. Once $M_{\pi}^{2}$ is fixed to its physical value no residual quenched chiral logarithms are left in the strong sector (with the mentioned exception of $\bar{q} q$ matrix elements). In Appendix B it is shown that the same situation occurs in the weak $\Delta I=1 / 2$ sector, where additional quenched chiral logarithms can be reabsorbed in the renormalization of the weak mass term.

\subsection{Scalar form factor}

The scalar form factor of the pion is defined by the matrix element of the $\bar{q} q$ density between two pion states

$$
\left\langle\pi^{i}\left(p^{\prime}\right)|\bar{q} q| \pi^{k}(p)\right\rangle=\delta^{i k} F_{S}(t),
$$

where $t=\left(p-p^{\prime}\right)^{2}$. In quenched CHPT the complete list of one-loop diagrams which give contribution to $F_{S}^{q}(t)$ are shown in Fig. 2.

An explicit calculation shows that the fermionic ghost loops do not fully cancel the corresponding meson loop diagrams. The reason for this mismatch is best understood within the quark-flow diagram picture. Here, the physical scalar source only couples to the quark lines and not to the ghost lines. The possible oneloop diagrams are the ones listed in Fig. [3. Diagram (b), where the scalar source is coupled to the internal disconnected closed quark line, has no correspondent ghost loop diagram: this is correct because the loop is not produced by the fermionic determinant, and must therefore be present also in the quenched approximation. 

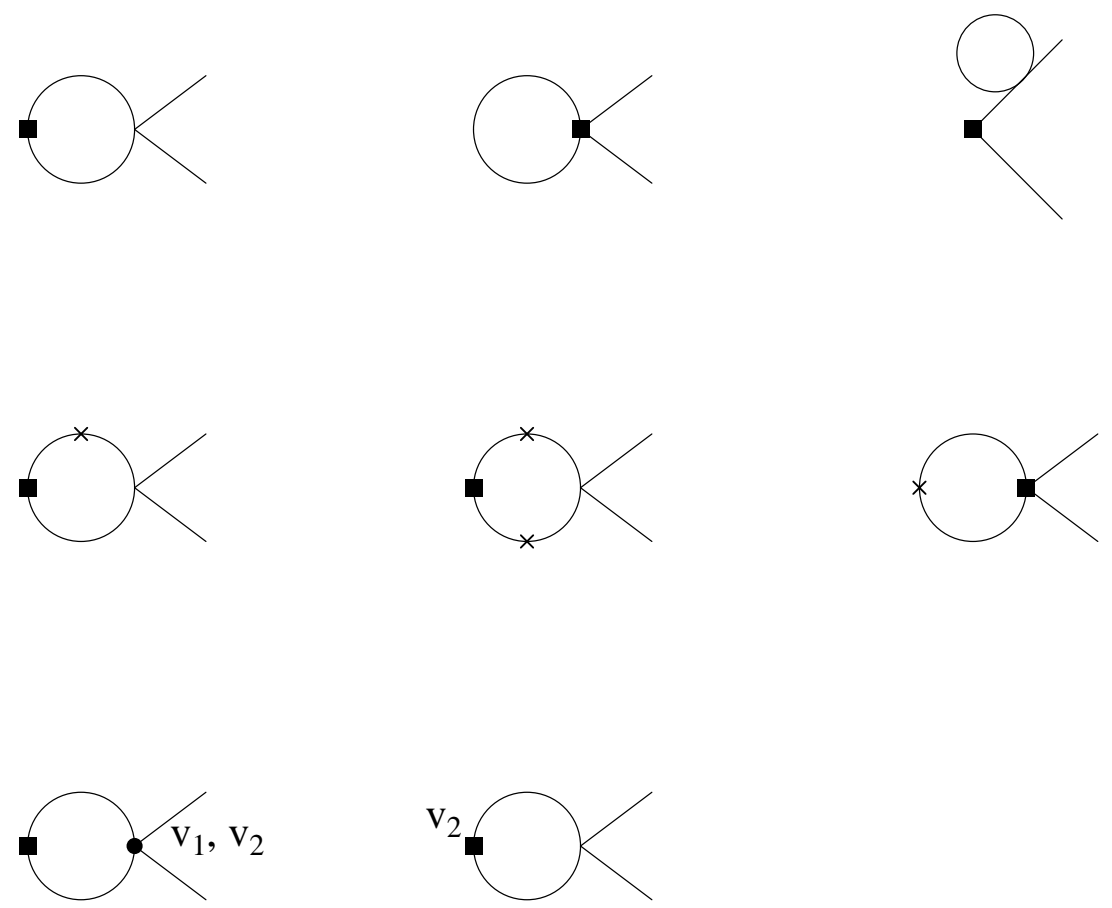

Figure 2: One-loop diagrams in quenched CHPT which contribute to $F_{S}(t)$ (the box stands for the scalar source insertion). . They are the "standard" meson loop diagrams (first line) to which one has to add the corresponding fermionic ghost loop diagrams, the singlet insertion diagrams (second line) and the diagrams with $v_{1}, v_{2}$ vertex insertions (third line).

The complete renormalized quenched scalar form factor can be written as follows

$$
\begin{aligned}
F_{S}^{q}(t)= & F_{S}^{q}(0)\left\{1+\frac{\bar{J}(t)}{F_{\pi}^{2}}\left[\frac{1}{2} \gamma_{4}^{q}\left(t-2 M_{\pi}^{2}\right)+\gamma_{3}^{q} M_{\pi}^{2}\right]+t \frac{\gamma_{4}^{q}}{32 \pi^{2} F_{\pi}^{2}}\left(\bar{l}_{4}^{q}-1\right)\right. \\
& -\frac{2}{3} \frac{M_{\pi}^{2}}{F_{\pi}^{2}} \bar{I}_{1}(t)\left(m_{0}^{2}-\alpha M_{\pi}^{2}\right)\left(1-\frac{2}{3} \alpha\right) \\
& \left.+\frac{2}{9} \frac{M_{\pi}^{2}}{F_{\pi}^{2}} \bar{I}_{2}(t)\left(m_{0}^{2}-\alpha M_{\pi}^{2}\right)^{2}\right\}+O\left(t^{2}\right)
\end{aligned}
$$

where the coefficients $\gamma_{i}^{q}$ have been defined in Eq. (4.14) and $F_{S}^{q}(0)$ is given by

$$
\begin{aligned}
F_{S}^{q}(0)= & 2 \bar{B}_{0}\left\{1-\frac{\left(m_{0}^{2}-\alpha M_{\pi}^{2}\right)}{48 \pi^{2} F_{\pi}^{2}}\left(1-\frac{2}{3} \alpha\right)+\frac{1}{9} \frac{\left(m_{0}^{2}-\alpha M_{\pi}^{2}\right)^{2}}{48 \pi^{2} F_{\pi}^{2} M_{\pi}^{2}}\right. \\
& \left.+\frac{M_{\pi}^{2}}{16 \pi^{2} F_{\pi}^{2}}\left[\gamma_{3}^{q}\left(\bar{l}_{3}^{q}-1\right)-\gamma_{4}^{q}\left(\bar{l}_{4}^{q}-1\right)\right]\right\} .
\end{aligned}
$$

Note that we are working in the degenerate mass case, so that no isospin breaking effect has been taken into account. In standard CHPT there is no isospin breaking 


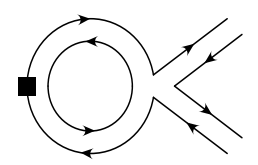

(a)

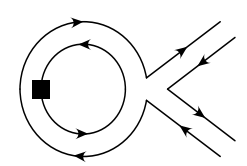

(b)

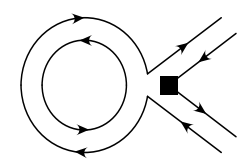

(c)

Figure 3: One-loop diagrams which contribute to $F_{S}(t)$ in the quark-flow diagram picture. Diagram $(b)$ can be present in the quenched approximation, while the others disappear.

correction to the scalar form factor at this order of the expansion. In passing, we state that also in the quenched case there is no isospin breaking contribution linear in $m_{u}-m_{d}$ to the pion scalar form factor, as it happens in CHPT, while an isospin breaking correction of order $\left(m_{u}-m_{d}\right)^{2}$ is produced via the $\left(\phi_{0}, \phi_{3}\right)$ mixing for neutral pions by the chiral invariant operator $P_{7}$ in Table $\mathbb{P}$.

The functions $\bar{J}(t), \bar{I}_{1}(t)$ and $\bar{I}_{2}(t)$ are finite and they are defined in Appendix G. The two functions $\bar{I}_{1}(t)$ and $\bar{I}_{2}(t)$ are peculiar of quenched CHPT. They will also appear in the $\pi \pi$ scattering amplitude, where we shall analyze in some details the various sicknesses of which they suffer. Here we used their low momentum expansion to define the scalar form factor at $t=0$.

The scalar form factor is a good example to analyze the modifications produced by the quenched approximation to an observable at the one loop level. First, the pion loops have been only partially cancelled, therefore the ordinary chiral logarithms and the one-loop function $\bar{J}(t)$ do appear in the same way as in standard CHPT, but with different coefficients (these coefficients may even vanish in particular cases, like $M_{\pi}$ and $F_{\pi}$ ). Second, quenched chiral logarithms appear at one loop, but they can be reabsorbed in the renormalization of the $B_{0}$ parameter, as we have demonstrated in the previous section. Besides quenched chiral logs, the remaining finite loop corrections arising from the anomalous singlet sector and proportional to $m_{0}^{2}$ are even more problematic, since they have negative chiral dimension, as anticipated in the general discussion above. It is a simple exercise to calculate the chiral dimension of the one loop diagram with two $m_{0}^{2}$ insertions on the two internal singlet lines (this is the central graph in the second line of Fig. 2): with respect to the tree level graph this has chiral dimension -2 . These corrections diverge in the chiral limit like an inverse pion mass squared, see Eq. (5.8). In fact, there is an infinite series of graphs that has the same chiral dimension: these graphs are obtained from this one by adding any even number of singlet lines (each one with one $m_{0}^{2}$ insertion) between the two vertices. Also the insertion of tadpoles and sunset diagrams with the maximum allowed number of $m_{0}^{2}$ insertions does not change the chiral dimension of the

\footnotetext{
${ }^{9}$ Note that also the neutral pion mass $M_{\pi^{0}}^{2}$ gets next-to-leading corrections of order $O\left(\left(m_{u}-m_{d}\right)^{2}\right)$ from $P_{7}$ in the quenched case.
} 
starting diagram. As far as we could see this series of graphs is also the one with the lowest chiral dimension for the scalar form factor. This example shows that despite the general formula (5.3) with $d=0$ vertices allowed, in the quenched case the chiral dimension of amplitudes is bounded from below.

It is also interesting to look at the slope of the scalar form factor at low momenta in the quenched case. This defines the scalar radius as follows

$$
F_{S}^{q}(t)=F_{S}^{q}(0)\left[1+\frac{t}{6}\left\langle r^{2}\right\rangle_{S}^{q}+O\left(t^{2}\right)\right]
$$

The scalar radius in the quenched approximation at one loop is given by:

$$
\begin{gathered}
\left\langle r^{2}\right\rangle_{S}^{q}=\frac{1}{16 \pi^{2} F_{\pi}^{2}}\left[\gamma_{3}^{q}+\gamma_{4}^{q}\left(3 \bar{l}_{4}^{q}-4\right)-\left(1-\frac{2}{3} \alpha\right) \frac{\left(m_{0}^{2}-\alpha M_{\pi}^{2}\right)}{3 M_{\pi}^{2}}\right. \\
\left.+\frac{4}{45} \frac{\left(m_{0}^{2}-\alpha M_{\pi}^{2}\right)^{2}}{M_{\pi}^{4}}\right] .
\end{gathered}
$$

In standard CHPT the scalar radius diverges in the chiral limit because of the presence of $t$ dependent chiral logarithms. It behaves like:

$$
\left\langle r^{2}\right\rangle_{S}=-\frac{3}{8 \pi^{2} F_{\pi}^{2}} \ln M_{\pi}^{2}+\ldots
$$

In the chiral limit the one-loop contribution to the quenched scalar radius diverges not just logarithmically as in the standard case, but like an inverse power of the pion mass:

$$
\left.\left\langle r^{2}\right\rangle_{S}^{q}\right|_{M_{\pi} \rightarrow 0} \sim \frac{1}{16 \pi^{2} F_{\pi}^{2}}\left[\frac{4}{45} \frac{m_{0}^{4}}{M_{\pi}^{4}}-\frac{1}{3}\left(1-\frac{2}{15} \alpha\right) \frac{m_{0}^{2}}{M_{\pi}^{2}}-3 \gamma_{4}^{q} \log M_{\pi}^{2}\right]+\ldots .
$$

The origin of this power-like divergence in the chiral limit is the same as that of the form factor at $t=0$. Here it is more severe simply because the definition of the radius implies a derivative with respect to $t$.

It is interesting to note that in quenched CHPT the Feynman-Hellman theorem [8, 15] does not hold:

$$
F_{S}^{q}(0) \neq \frac{\partial M_{\pi}^{2}}{\partial m_{q}}
$$

as one can easily verify by comparing Eq. (5.5) and Eq. (5.8). The origin of the violation of this theorem is in the presence of diagram (b) of Fig. 3 in the quenched scalar form factor. This graph cannot be obtained taking a derivative with respect to $m_{q}$ of $M_{\pi}^{2}$, since the quark loop is not present in $M_{\pi}^{2}$ and cannot be resurrected by a derivative. 

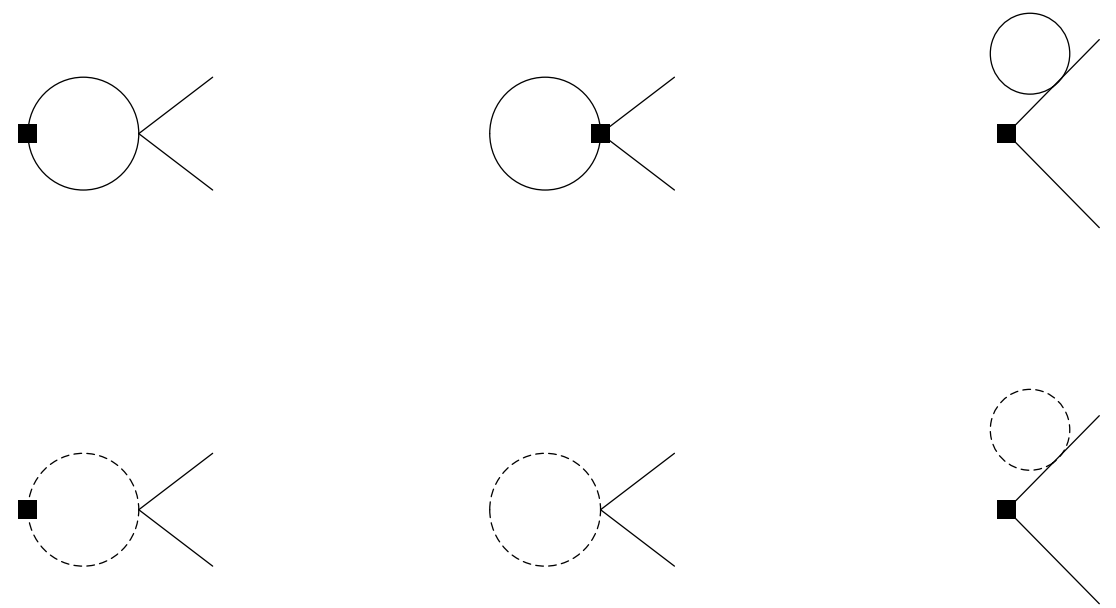

Figure 4: One-loop diagrams in quenched CHPT that contribute to $F_{V}^{q}(t)$ (the box stands for the vector source insertion). They are the "standard" meson loop diagrams (first line) and the corresponding fermionic ghost loop diagrams (second line). No singlet component can run in the loop.

\subsection{Vector form factor}

The vector form factor of the pion is defined in terms of the matrix element of the vector current $V_{\mu}^{k}=\bar{q} \gamma_{\mu} \frac{\lambda^{k}}{2} q$ between two pion states:

$$
\left\langle\pi^{i}\left(p^{\prime}\right)\left|V_{\mu}^{k}\right| \pi^{l}(p)\right\rangle_{q}=i \epsilon^{i k l}\left(p_{\mu}+p_{\mu}^{\prime}\right) F_{V}^{q}(t)
$$

where $t=\left(p-p^{\prime}\right)^{2}$. The divergent contributions to $F_{V}^{q}(t)$ can be derived from the expression (3.21) of the quenched generating functional in the usual way. It is an easy exercise to show that these contributions are zero. In fact, the only chiral invariant which can give corrections at order $p^{4}$ is the operator number 9 in the list of Table 1, which has no divergent term in the quenched limit. In a Feynman diagram approach the graphs which contribute to one loop are shown in Fig. 4 . The complete calculation gives zero, because of the systematic cancellation of each pion loop with the corresponding ghost loop. In addition, since no singlet component can run in the loop, there is no extra contribution coming from the anomalous singlet sector. The quenched vector form factor for $N=2$ can be written as follows

$$
F_{V}^{q}(t)=1-\frac{l_{6}^{q}}{F_{\pi}^{2}} t+O\left(p^{4}\right)
$$

where the finite counterterm $l_{6}^{q}$ is defined in Table 1 and Eq. (4.10). Again, no isospin breaking effects have been taken into account. In standard CHPT the Ademollo-Gatto theorem [16] guarantees that they are absent at this order. In quenched CHPT the theorem is also valid. Note that the counterterm $P_{7}$ cannot contribute at all to the vector current matrix element, while the new chiral invariant term $\left\langle u_{\mu}\right\rangle\left\langle u^{\mu} \chi_{+}\right\rangle$induced by the dynamical singlet component 


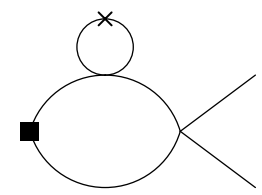

$\delta \mathrm{D}_{\mathrm{g}}=2$

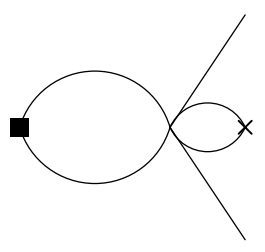

$\delta \mathrm{D}_{\mathrm{g}}=2$

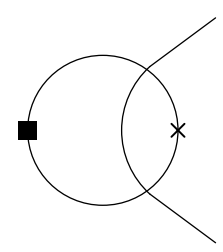

$\delta \mathrm{D}_{\mathrm{g}}=2$

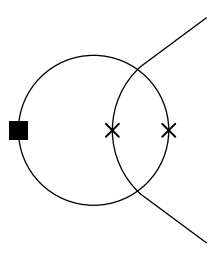

$\delta \mathrm{D}_{\mathrm{g}}=0$

Figure 5: Two-loop diagrams in quenched CHPT with the $m_{0}^{2}$ singlet vertex $(\times)$ insertions that give divergent contributions to the e.m. charge radius in the chiral limit. They are tadpoles, which generate quenched chiral logs and the fish diagrams that also generate power-like divergences. For each diagram the chiral dimension respect to the tree level is given.

gives $O\left(\left(m_{u}-m_{d}\right)^{2}\right)$ corrections to the decay amplitude $\pi^{+} \rightarrow \pi^{0} e \nu$ via the $\left(\phi_{0}, \phi_{3}\right)$ mixing.

Since the vector form factor does not receive contributions from singlets running inside the loop at the one-loop level, it does not show any divergence in the chiral limit. The situation however changes at two loops, where we have among others the graphs shown in Fig. 5. The most dangerous graph is the fish diagram with two $m_{0}^{2}$ insertions (the last of Fig. 5) which has chiral dimension zero respect to the tree level. Again this is only the first example of a full series of graphs which have the same chiral dimension: they are obtained from the starting one by inserting any even number of singlet lines between the same two vertices as those of the two-loop fish diagram, or tadpoles and sunset diagrams all with the maximum allowed number of $m_{0}^{2}$ insertions. In this case there are no graphs which are more singular than those in the chiral limit.

The low energy representation of $F_{V}^{q}(t)$ also determines the electromagnetic charge radius of the pion in the quenched approximation

$$
F_{V}^{q}(t)=1+\frac{t}{6}\left\langle r^{2}\right\rangle_{V}^{q}+O\left(t^{2}\right)
$$

In standard CHPT the presence of $t$ dependent chiral logarithms makes the electromagnetic charge radius diverge in the chiral limit [8]

$$
\left\langle r^{2}\right\rangle_{V}=-\frac{1}{16 \pi^{2} F_{\pi}^{2}} \log M_{\pi}^{2}+\ldots
$$

The divergence of the electromagnetic charge radius in full QCD can be understood in a physically intuitive way. The charge distribution is cut off by the Yukawa potential $\sim e^{-M_{\pi} r}$ at large distances. In the chiral limit $M_{\pi}$ goes to zero and the Yukawa potential is no more effective, the charge distribution falls off like a power of the distance and the charge radius becomes infinite. The charge distribution of the pion cloud surrounding any particle gets modified by quenching. As a consequence, the behaviour of the charge radius in the chiral limit is 


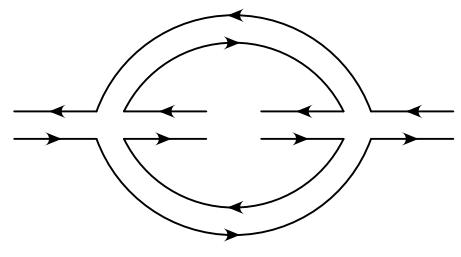

(a)

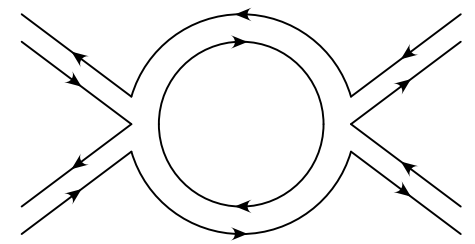

(b)

Figure 6: Two examples of pion loop graphs contributing to $\pi \pi$ scattering in the quark-flow diagram picture (all lines are quark lines). Diagram (a) does not contain quark loops, whereas diagram (b) does.

modified. In the quenched case the one-loop contribution gives

$$
\left\langle r^{2}\right\rangle_{V}^{q}=-\frac{6 l_{6}^{q}}{F_{\pi}^{2}}
$$

which stays finite in the chiral limit. The situation changes at two loops and higher: the graphs that we have discussed above, which have chiral dimension zero with respect to the tree level (like the two-loop fish diagram), do generate power-like divergences in the chiral limit. At two loops we are going to have a behaviour like:

$$
\left.\left\langle r^{2}\right\rangle_{V}^{q 2 \text { loop }}\right|_{M_{\pi} \rightarrow 0} \sim \frac{1}{\left(16 \pi^{2} F_{\pi}^{2}\right)^{2}}\left(d_{1} \frac{\left(m_{0}^{2} / N_{c}\right)^{2}}{M_{\pi}^{2}}+d_{2} \frac{m_{0}^{2}}{N_{c}} \ln M_{\pi}^{2}\right),
$$

where presumably also at this order the chiral logs could be reabsorbed in the renormalization of some order $p^{4}$ constants.

\subsection{The $\pi \pi$ scattering amplitude}

The $\pi \pi$ scattering amplitude is another example of an observable where one can find all the typical effects of quenching. Moreover it is an interesting quantity by itself since a comparison of the prediction for the two $S$-wave scattering lengths with existing lattice calculations [17] is possible.

The presence of "standard" chiral logs even in the quenched theory has to be interpreted as due to diagrams with pion loops that do not contain quark loops. For the $\pi \pi$ scattering amplitude an example is given in Fig. 6. The one-loop contributions to the $\pi \pi$ scattering amplitude in quenched CHPT come from the diagrams shown in Fig. 7 . The scattering amplitude at tree level is the same as in standard CHPT

$$
A^{\text {tree }}(s, t, u)=\frac{s-M^{2}}{F^{2}}
$$

where $M$ and $F$ are the bare pion mass and decay constant. The renormalized scattering amplitude in quenched CHPT and in the two degenerate flavour case 


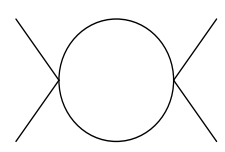

s-channel

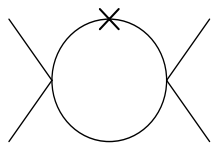

s-channel

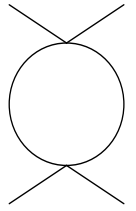

$\mathrm{t}-$,u-channel

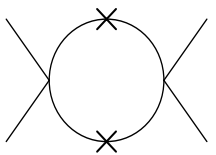

s-channel
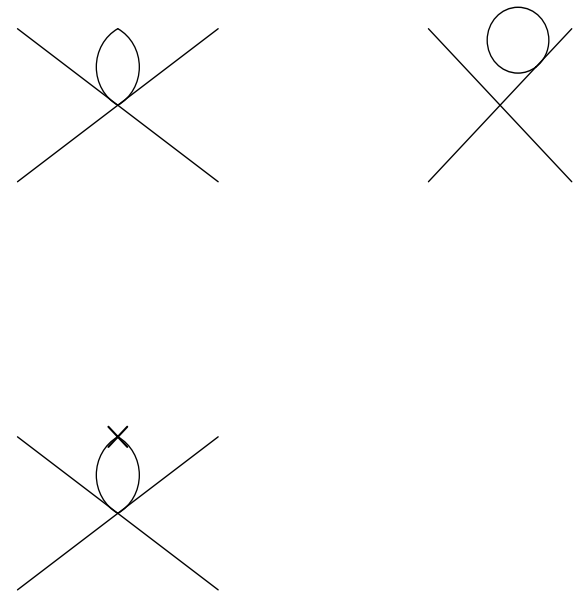

\section{$\mathrm{v}_{1,2}$}

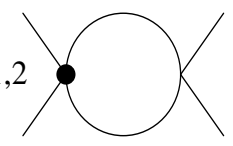

\section{s-channel}

Figure 7: One-loop diagrams in quenched CHPT which contribute to the $\pi \pi$ scattering amplitude in the two degenerate flavour case. They are the "standard" meson loop diagrams (first line) to which one has to add the corresponding fermionic ghost loop diagrams, the singlet vertex $(\times)$ insertion diagrams (second line) and the diagrams with one $v_{1}, v_{2}$ vertex insertion (third line).

can be written as follows

$$
A(s, t, u)=\frac{s-M_{\pi}^{2}}{F_{\pi}^{2}}+B(s, t, u)+C(s, t, u)+O\left(p^{6}\right),
$$

where

$$
\begin{aligned}
B(s, t, u)= & \frac{\bar{J}(s)}{4 F_{\pi}^{4}}\left\{s^{2}-16 M_{\pi}^{2} v_{1}\left(s-2 M_{\pi}^{2}\right)+4 M_{\pi}^{4}\left(\gamma_{3}^{q}-1\right)\right\} \\
& +\frac{1}{4 F_{\pi}^{4}}\left\{\bar{J}(t)\left(t-2 M_{\pi}^{2}\right)^{2}+\bar{J}(u)\left(u-2 M_{\pi}^{2}\right)^{2}\right\} \\
& +I_{1}(s) \frac{2 M_{\pi}^{4}}{3 F_{\pi}^{4}}\left(m_{0}^{2}-\alpha M_{\pi}^{2}\right)\left(\frac{2}{3} \alpha-1\right)+I_{2}(s) \frac{2 M_{\pi}^{4}}{9 F_{\pi}^{4}}\left(m_{0}^{2}-\alpha M_{\pi}^{2}\right)^{2},
\end{aligned}
$$




$$
\begin{aligned}
C(s, t, u)= & \frac{1}{128 \pi^{2} F_{\pi}^{4}}\left\{s^{2}\left(2 \bar{l}_{1}^{q}+\bar{l}_{2}^{q}-3\right)+(t-u)^{2}\left(\bar{l}_{2}^{q}-1\right)\right. \\
& +8 s M_{\pi}^{2}\left[1-\bar{l}_{1}^{q}+\gamma_{4}^{q}\left(\bar{l}_{4}^{q}-1\right)\right] \\
& \left.+8 M_{\pi}^{4}\left[\bar{l}_{1}^{q}-1+\gamma_{3}^{q}\left(\bar{l}_{3}^{q}-1\right)-2 \gamma_{4}^{q}\left(\bar{l}_{4}^{q}-1\right)\right]\right\} .
\end{aligned}
$$

For a definition of the functions $\bar{J}\left(q^{2}\right), I_{1}\left(q^{2}\right)$ and $I_{2}\left(q^{2}\right)$ see Appendix $\mathbf{Q}$. The functions $I_{1}(s)$ and $I_{2}(s)$ arise from diagrams with one and two $m_{0}^{2}$ insertions on the two internal singlet lines in the $s$-channel respectively (see Fig. 7). Note that everything is expressed in terms of the renormalized squared pion mass $M_{\pi}^{2}$ given by Eq. (5.5) and $F_{\pi}=\bar{F}$. Note also that any dependence upon quenched chiral logarithms has been again reabsorbed in the $\bar{B}_{0}$ parameter contained in the renormalized pion mass, as expected. The function $C(s, t, u)$ contains only polynomial contributions, while the invariant function $B(s, t, u)$ is the quenched analogue of the unitarity correction to the scattering amplitude in ordinary CHPT. It is important to note that unitarity is destroyed by the quenched approximation: the structure of the cuts in the one-loop amplitude is not related via unitarity to the real part of the tree level amplitude. Moreover, one can easily verify that the Fermi-Watson theorem, which relates, e.g., the imaginary part of the vector and scalar form factors to those of the corresponding partial waves of $\pi \pi$ scattering, is not valid in this case.

In this particular example the violation of unitarity is also immediately seen in the presence of the finite functions $I_{1}\left(q^{2}\right)$ and $I_{2}\left(q^{2}\right)$, which are not generated in ordinary CHPT. They have a nonzero imaginary part for $s \geq 4 M^{2}$ that has a singularity at $s=4 M^{2}$ (of the type $\left(s-4 M^{2}\right)^{-1 / 2}$ and $\left(s-4 M^{2}\right)^{-3 / 2}$, respectively, see Appendix Q), which is a pure quenching artifact. These singularities have been already identified in [12, 18]. Here we have rederived them in the $\alpha \neq 0$ case and inserted in the complete formula for the amplitude.

Interesting quantities to be extracted from the $\pi \pi$ scattering amplitude are the $S$-wave scattering lengths. In Ref. [10] we calculated the coefficients of the chiral logarithms which arise in the quenched case and made the comparison with standard CHPT. Here we give the complete expression of the $S$-wave scattering lengths in the isospin $I=0,2$ channels to one loop and comment on the anomalous behaviour of the isospin amplitude in the $I=0$ channel (which was already remarked in Ref. [12]). The $I=0,2$ amplitudes are expressed in terms of the invariant amplitude $A(s, t, u)$ as follows

$$
\begin{aligned}
& T^{0}(s, t)=3 A(s, t, u)+A(t, u, s)+A(u, s, t), \\
& T^{2}(s, t)=A(t, u, s)+A(u, s, t) .
\end{aligned}
$$

The pion scattering lengths $a_{l}^{I}$ for a given isospin $I$ and angular momentum $l$ are defined by the behaviour of the partial wave amplitudes near threshold

$$
\operatorname{Re} t_{l}^{I}(s)=q^{2 l}\left\{a_{l}^{I}+q^{2} b_{I}^{l}+O\left(q^{4}\right)\right\} \text {, }
$$


which enter the expansion in partial waves of the isospin amplitude

$$
T^{I}(s, t)=32 \pi \sum_{l=0}^{\infty}(2 l+1) P_{l}(\cos \theta) t_{l}^{I}(s) .
$$

For more details about the notation we refer the reader to Ref. [8]. The scattering amplitude in the $I=0$ channel contains the amplitude $A(s, t, u)$ and therefore in the quenched case acquires a sick threshold behaviour due to the presence of functions $I_{1}(s)$ and $I_{2}(s)$. These functions do not contribute to the $I=2$ amplitude. On the other hand the divergences at threshold present in the infinite volume case show up as "enhanced" finite volume corrections to the Lüscher formula [19], that is used on the lattice to extract the scattering lengths; these finite volume corrections have been studied in Ref. [12]. We can formally define the quenched $I=0 S$-wave scattering length $a_{0}^{0}$ as the coefficient of the $\left(q^{2}\right)^{0}$ term in the expansion of the real part of the isospin amplitude $T^{0}(s, t)$ in partial waves. This gives us an idea of the size of normal one-loop corrections to the scattering length. The present definition is also equivalent to the one adopted in Ref. [12 in the analysis of the finite volume corrections. The quenched $S$-wave scattering length in the $I=2$ channel $a_{0}^{2}$ is defined in the usual way. For the complete renormalized $S$-wave "quenched scattering lengths" at one loop we find:

$$
\begin{aligned}
\frac{32 \pi F_{\pi}^{2}}{M_{\pi}^{2}} a_{0}^{0}= & 7+\frac{M_{\pi}^{2}}{16 \pi^{2} F_{\pi}^{2}}\left\{7+5\left(\bar{l}_{1}^{q}+2 \bar{l}_{2}^{q}\right)+\gamma_{3}^{q}\left(5 \bar{l}_{3}^{q}+1\right)+2 \gamma_{4}^{q}\left(\bar{l}_{4}^{q}-1\right)-48 v_{1}\right\} \\
& -\frac{\left(m_{0}^{2}-\alpha M_{\pi}^{2}\right)}{48 \pi^{2} F_{\pi}^{2}}\left(\frac{2}{3} \alpha-1\right)+\frac{5}{9} \frac{\left(m_{0}^{2}-\alpha M_{\pi}^{2}\right)^{2}}{48 \pi^{2} M_{\pi}^{2} F_{\pi}^{2}} \\
\frac{32 \pi F_{\pi}^{2}}{M_{\pi}^{2}} a_{0}^{2}= & -2+\frac{M_{\pi}^{2}}{16 \pi^{2} F_{\pi}^{2}}\left\{2\left(\bar{l}_{1}^{q}+2 \bar{l}_{2}^{q}-1\right)+2 \gamma_{3}^{q}\left(\bar{l}_{3}^{q}-1\right)-4 \gamma_{4}^{q}\left(\bar{l}_{4}^{q}-1\right)\right\} \\
& +\frac{\left(m_{0}^{2}-\alpha M_{\pi}^{2}\right)}{24 \pi^{2} F_{\pi}^{2}}\left(\frac{2}{3} \alpha-1\right)+\frac{2}{9} \frac{\left(m_{0}^{2}-\alpha M_{\pi}^{2}\right)^{2}}{48 \pi^{2} M_{\pi}^{2} F_{\pi}^{2}} .
\end{aligned}
$$

The renormalized quenched scattering lengths depend upon four counterterms $\bar{l}_{1}^{q}, \ldots \bar{l}_{4}^{q}$ and the parameters of the anomalous singlet sector at leading order, $m_{0}^{2}$, $\alpha, v_{1}$ and $v_{2}$. The counterterms $\bar{l}_{i}^{q}$ carry the chiral logarithms $\bar{l}_{i}^{q}=-\log m+\ldots$. In ordinary CHPT the chiral logarithms are largely dominant in the one-loop corrections to the $S$-wave scattering lengths at the renormalization scale $\mu=1$ $\mathrm{GeV}$ [20]. Here the main unknown is the value of the parameters $v_{1}, v_{2}$ of the singlet sector. The singlet parameters $m_{0}$ and $\alpha$ can be extracted from lattice calculations. Favoured values are listed e.g. in Ref. [21. With these values at hand we can do the following numerical exercise. Let us disregard for the moment the parameters $v_{1}$ and $v_{2}$ and limit the analysis to the contributions that are reasonably expected to be the dominant ones: 1) the singlet corrections 
in $m_{0}$ and $\alpha$ and 2) the standard chiral logarithms. With the definitions

$$
\delta=\frac{m_{0}^{2}}{48 \pi^{2} F_{\pi}^{2}}, \quad \epsilon=\frac{M_{\pi}^{2}}{48 \pi^{2} F_{\pi}^{2}}, \quad \bar{\delta}=\frac{m_{0}^{2}-\alpha M_{\pi}^{2}}{48 \pi^{2} F_{\pi}^{2}}=\delta-\alpha \epsilon,
$$

the leading contributions to the scattering lengths are as follows:

$$
\begin{aligned}
& \frac{32 \pi F_{\pi}^{2}}{M_{\pi}^{2}} a_{0}^{0}=7-\left(\frac{2}{3} \alpha-1\right) \bar{\delta}+\frac{5}{9} \frac{\bar{\delta}^{2}}{\epsilon}-66 \epsilon \ln \frac{M_{\pi}^{2}}{\mu^{2}}+\ldots, \\
& \frac{32 \pi F_{\pi}^{2}}{M_{\pi}^{2}} a_{0}^{2}=-2+\left(\frac{2}{3} \alpha-1\right) 2 \bar{\delta}+\frac{2}{9} \frac{\bar{\delta}^{2}}{\epsilon}-12 \epsilon \ln \frac{M_{\pi}^{2}}{\mu^{2}}+\ldots .
\end{aligned}
$$

For the numerical calculations we use $F_{\pi}=93 \mathrm{MeV}, \delta=0.15$ and $\alpha=0.6$ and vary the pion mass between its physical value $M_{\pi}=140 \mathrm{MeV}$, and $M_{\pi}=$ $600 \mathrm{MeV}$, which is presumably already outside a reasonable range of validity for ordinary CHPT. The chiral $\log$ is evaluated at $\mu=1 \mathrm{GeV}$. The numerical results are given in Tables 2 and 3 for the $I=0$ and $I=2$ scattering lengths respectively. We note that at the physical value of the pion mass the $\bar{\delta}^{2} / \epsilon$ term is largely dominant in both cases: the divergence in the chiral limit produced by quenching is already felt at the physical pion mass. This also means that the whole framework is not very reliable in this range, since also higher loop effects may produce modifications of the same chiral order (higher powers of $\delta$ with the same $1 / \epsilon$ in front). At larger values of the pion mass, which are those typically used in lattice calculations, the situation changes and the standard chiral logarithms become dominant, as it happens in standard CHPT.

\begin{tabular}{|c|c|c|c|c|}
\hline$M_{\pi}(\mathrm{MeV})$ & tree & $\bar{\delta}$ & $\bar{\delta}^{2} / \epsilon$ & $\epsilon \ln M_{\pi}^{2}$ \\
\hline & & & & \\
140 & 7 & 0.09 & 2.5 & 1.2 \\
300 & 7 & 0.08 & 0.47 & 3.5 \\
600 & 7 & 0.06 & 0.06 & 5.9 \\
\hline
\end{tabular}

Table 2: Numerical values of the leading contributions to $a_{0}^{0}$ quenched up to one loop for $M_{\pi}=140,300,600 \mathrm{MeV}$, according to Eq. (5.29).

This picture, although at a semiquantitative level, suggests that quenched lattice calculations of the $S$-wave scattering lengths with a moderately high pion mass (like the ones in Ref. [17]), should not be too far from those predicted by full CHPT. This conclusion is based on two observations: first the standard chiral logarithms start soon to be dominant with respect to the dangerous quenching effects, and second their coefficient happens not to be substantially changed by quenching [10]. The comparison between the standard CHPT prediction at one [8] and two loops [22], and the lattice calculation [17], has been made in Ref. [23]. 


\begin{tabular}{|c|c|c|c|c|}
\hline$M_{\pi}(\mathrm{MeV})$ & tree & $\bar{\delta}$ & $\bar{\delta}^{2} / \epsilon$ & $\epsilon \ln M_{\pi}^{2}$ \\
\hline & & & & \\
140 & -2 & -0.18 & 1.0 & 0.23 \\
300 & -2 & -0.16 & 0.19 & 0.63 \\
600 & -2 & -0.12 & 0.02 & 1.1 \\
\hline
\end{tabular}

Table 3: Numerical values of the leading contributions to $a_{0}^{2}$ quenched up to one loop for $M_{\pi}=140,300,600 \mathrm{MeV}$ according to Eq. (5.29)

\section{Summary and conclusions}

In this paper we have analyzed the quenched version of Chiral Perturbation Theory at the one-loop level. We have calculated the one-loop ultraviolet divergences of the theory at the level of the generating functional, and shown how one can reabsorb all those divergences by a proper definition of the counterterms. We have shown that even in the presence of the anomalous singlet sector the ultraviolet divergent part of the quenched generating functional can be calculated in closed form. We have closely followed the notation and methods of standard CHPT [8] in order to identify as clearly as possible the changes produced by the quenched approximation in the formulation of the effective theory.

We have found a systematic cancellation of the flavour-number dependent terms inside the divergent part of the generating functional to one loop. As we anticipated in Ref. [10] the complete $N$-independence of quenched CHPT is welcome, since it shows that we understand the differences between standard CHPT and its quenched version. Let us recall that the calculation of the divergences to one loop in CHPT produces explicit $N$ dependence, in three different powers: $N$, $1 / N$ and $1 / N^{2}$. The terms linear in $N$ must be generated at the quark level by virtual quark loops: therefore they must be absent in the quenched theory. The terms with inverse powers of $N$ are generated by the decoupling of the singlet field from the octet of the Goldstone bosons. Since the decoupling does not take place in the quenched theory, also the inverse powers of $N$ disappear in qCHPT to one loop. A posteriori one could say that the changes that lead from standard CHPT to its quenched version could have been guessed by simply looking at the $N$ dependence of the generating functional to one loop. In fact this can still be done in other sectors of the effective theory that have not been fully analyzed yet. We give one example of this in Appendix B, where we study the one-loop divergences in the sector of the on-shell non-leptonic weak interactions. 
The quenched approximation produces a double pole in the singlet two-point function, which however is not allowed in a consistent quantum field theory, and is therefore the source of many sicknesses of quenched CHPT. As was shown already in Refs. [2, 3], one of the consequences of this double pole is the appearance of a new kind of chiral logarithms in the one-loop corrections. Together with the standard $M^{2} \ln M^{2}$ chiral logarithms, qCHPT has corrections of the form $m_{0}^{2} \ln M^{2}$, which diverge in the chiral limit. The complete calculation of all the ultraviolet one-loop divergences in the generating functional, has shown that the quenched chiral logs can be accounted for via a renormalization of the lowest order constant $B_{0}$ (which is proportional to the quark condensate). As a consequence, the renormalized $\bar{B}_{0}$ parameter diverges in the chiral limit, while the renormalized pion mass $M_{\pi}^{2}=2 \bar{B}_{0} m_{q}$ does not. The use of the renormalized pion mass to express any other observable makes the quenched chiral logs disappear at one loop, with the only exception of $\bar{q} q$ matrix elements, that are proportional to the renormalized $\bar{B}_{0}$ parameter alone. Hence, $\bar{q} q$ matrix elements remain the unique place for discovering the presence of quenched chiral logs in quenched lattice calculations within the strong sector.

The double pole in the singlet two-point function also changes the standard chiral power counting for which diagrams with a higher number of loops are of higher chiral order. In the quenched case one may have graphs with any number of loops with the same chiral dimension, and the chiral order of an amplitude is no more constrained to be positive: as a consequence, quenched CHPT has power-like divergences in the chiral limit. These divergences are in principle a very serious problem of the theory, although they seem to be a unavoidable consequence of the quenched approximation. Since the graphs that have negative chiral dimension are also ultraviolet finite, their study requires the calculation of the UV finite part of the loop corrections. We have shown how they arise within the generating functional approach; at one-loop and at order $\Phi^{4}$ they are given in Eq. (3.22). We have therefore analyzed some physical quantities at one loop in the case of two degenerate light flavours: the scalar quark condensate, the pion mass, the scalar and vector form factors of the pion and the $\pi \pi$ scattering amplitude. This has given us the possibility to discuss in detail the changes induced by quenching in the UV finite part of the one-loop corrections. The main changes can be summarized by saying that unitarity is not satisfied anymore, and that the double pole in the singlet two-point function produces singularities in the chiral limit, and also unphysical singularities at threshold.

The differences between CHPT and its quenched version are rather well understood, as the study of the flavour-number dependence of the generating functional at one-loop also shows. The presence of the double pole in the singlet two-point function is also a rather direct consequence of the quenched approximation. This 
double pole has dramatic effects on the effective theory. However, it looks plausible that despite all these inconsistencies (or maybe because of them) quenched CHPT is the right tool to understand the effects of quenching in actual lattice calculations. The crucial check will be a detailed comparison of qCHPT predictions with the quark mass dependence of various quenched quantities on the lattice, and especially of the way they approach the chiral limit. We expect that further investigations in this direction will answer these questions.

\section{Acknowledgements}

We thank Roberto Petronzio and Juerg Gasser for many enlightening discussions, Gerhard Ecker and Joachim Kambor for informative discussions about the weak non-leptonic sector. This work has been supported by Schweizerisches Nationalfonds and by the HCM, EEC-Contract No. CHRX-CT920026 (EURODAФNE). 


\section{A Divergences proportional to $m_{0}^{2}$}

In this appendix we try to explain in a simple way why the divergence proportional to $m_{0}^{2}$ is given by the chiral invariant term $\left\langle\chi_{+}\right\rangle$. We start from the observation that the double pole in the singlet propagator can be expressed with a derivative of a normal propagator:

$$
\frac{m_{0}^{2}}{\left(M^{2}-p^{2}\right)^{2}}=-m_{0}^{2} \frac{\partial}{\partial M^{2}} \frac{1}{M^{2}-p^{2}} .
$$

In fact in the quenched case one is keeping only the first two terms in a Taylor series:

$$
\frac{1}{M_{\eta^{\prime}}^{2}-p^{2}}=\frac{1}{M^{2}-p^{2}}-\left(M_{\eta^{\prime}}^{2}-M^{2}\right) \frac{1}{\left(M^{2}-p^{2}\right)^{2}}+\ldots,
$$

where $m_{0}^{2}=M_{\eta^{\prime}}^{2}-M^{2}$. The divergences proportional to $m_{0}^{2}$ arise from this Taylor expansion of the propagator. Before the expansion, singlet loops give two type of divergent contributions:

$$
\frac{1}{i} \int \frac{d^{d} p}{(2 \pi)^{d}} \frac{1}{M_{\eta^{\prime}}^{2}-p^{2}}, \text { and } \frac{1}{i} \int \frac{d^{d} p}{(2 \pi)^{d}} \frac{1}{M_{\eta^{\prime}}^{2}-p^{2}} \frac{1}{M_{\eta^{\prime}}^{2}-(p-q)^{2}} .
$$

The divergence proportional to $m_{0}^{2}$ is obtained expanding the singlet propagator inside the loop integrals and taking only the second term in the expansion. It is clear that only the tadpole produces a divergence:

$$
\frac{1}{i} \int \frac{d^{d} p}{(2 \pi)^{d}} \frac{1}{M_{\eta^{\prime}}^{2}-p^{2}} \rightarrow \frac{1}{i} \int \frac{d^{d} p}{(2 \pi)^{d}} \frac{-m_{0}^{2}}{\left(M^{2}-p^{2}\right)^{2}}=-m_{0}^{2} J(0) .
$$

The chiral structure of the term proportional to the tadpole is very easily identified, and is given by the term proportional to $\xi_{0}^{2}$ after expanding the action in fluctuations around the classical solution:

$$
\mathcal{L}_{2}=\frac{F^{2}}{4}\left\langle u_{\mu} u^{\mu}+\chi_{+}\right\rangle \rightarrow \frac{F^{2}}{4}\left\{-\xi_{0}\left(\square+M_{\eta^{\prime}}^{2}\right) \xi_{0}-\xi_{0}^{2}\left(\left\langle\chi_{+}\right\rangle-2 N M^{2}\right)\right\} .
$$

Obviously, the tadpole is generated by the contraction of the two $\xi_{0}$ 's in the last term of Eq. (A.5), and therefore the divergence is proportional to $\left\langle\chi_{+}\right\rangle$.

In summary: the divergence proportional to $m_{0}^{2}$ must be, for dimensional reasons, a chiral term of order $p^{2}$. This divergence comes out from a tadpole through a derivative with respect to $M_{\eta^{\prime}}^{2}$. In the tadpole the vertex is a chiral invariant of order $p^{2}$. The simplified recipe to derive the form of the divergent term proportional to $m_{0}^{2}$ amounts to determining the chiral invariant that is proportional to $\xi_{0}^{2}$ after expanding the CHPT Lagrangian in fluctuations around the classical solution.

The recipe applies in the same way to other sectors. We will give an example below for the non-leptonic weak interactions sector. 


\section{B Non-leptonic weak interactions}

In this appendix we show how one can derive the structure of the divergences in the quenched case just by looking at the $N$ dependence of the divergences of the standard CHPT case. We will consider the weak octet Lagrangian which contributes to the non-leptonic weak interactions with $\Delta I=1 / 2$. The structure of the divergences in standard CHPT has been given by Kambor, Missimer and Wyler [24], and then expressed in terms of a minimal basis by Ecker, Kambor and Wyler [25] for on-shell processes. We will use the basis given in the latter Reference.

Let us recall here some basic notation. In this sector the lowest order Lagrangian is given by:

$$
\mathcal{L}_{W 2}^{8}=c_{2}\left\langle\Delta u_{\mu} u^{\mu}\right\rangle+c_{5}\left\langle\Delta \chi_{+}\right\rangle,
$$

where $\Delta=u \lambda_{6} u^{\dagger}$, and $c_{2,5}$ are low energy constants. The $c_{5}$ term can be omitted for on-shell processes, as it can be transformed away by a field redefinition. We will not consider it anymore in what follows. Since the above-mentioned calculations of the divergences were made for $N=3$ we have redone the calculation of the divergences for $N$ generic. Our result for the divergent part of the one-loop generating functional, reads as follows:

$$
\begin{aligned}
Z_{\text {one loop }}^{8}= & -\frac{\mu^{d-4}}{16 \pi^{2}} \frac{1}{d-4} \frac{c_{2}}{F_{\pi}^{2}} \int d x L_{\text {div }}^{8}+\text { finite terms } \\
L_{\text {div }}^{8}= & W_{4}+\frac{1}{2} W_{6}-\frac{3}{4} W_{7}+\frac{1}{4} W_{8}-\frac{1}{2} W_{11} \\
+ & \frac{1}{N}\left[2 W_{10}+W_{12}-2 W_{21}-2 W_{22}+W_{36}-\frac{2}{N} W_{11}\right] \\
+ & N\left[\frac{2}{3} W_{1}-\frac{1}{6} W_{2}+\frac{1}{4} W_{5}+\frac{1}{4} W_{9}-\frac{1}{4} W_{12}+\frac{1}{12} W_{14}+\frac{1}{6} W_{15}-\frac{1}{12} W_{16}\right. \\
& \quad-\frac{1}{24} W_{18}-\frac{5}{12} W_{19}+\frac{1}{4} W_{20}+\frac{1}{2} W_{21}+\frac{1}{2} W_{22}+\frac{1}{6} W_{25}-\frac{1}{4} W_{26} \\
& \left.+\frac{1}{24} W_{27}-\frac{1}{4} W_{36}-\frac{1}{24} W_{37}-\frac{1}{4} W_{38}\right] .
\end{aligned}
$$

With $W_{i}$ we have indicated the operators of order $p^{4}$ given in Ref. [25]. Here we have one more:

$$
W_{38}=\left\langle\Delta u_{\mu} \chi_{+} u^{\mu}\right\rangle
$$

that for $N=3$ is linearly dependent on the other operators thanks to the trace identity coming from the Cayley-Hamilton theorem: $W_{38}=-W_{5}+W_{6}+1 / 2 W_{7}+$ $W_{8}$.

According to the rules we have found in the strong sector, the divergences of the octet weak sector for on-shell processes in the quenched case become:

$$
L_{\mathrm{div}}^{8 q}=W_{4}+\frac{1}{2} W_{6}-\frac{3}{4} W_{7}+\frac{1}{4} W_{8}-\frac{1}{2} W_{11} .
$$


For the sake of clarity we list the definitions of the $W_{i}$ terms needed here:

$$
\begin{aligned}
& W_{4}=\left\langle\Delta u_{\mu}\right\rangle\left\langle u^{\mu} u_{\nu} u^{\nu}\right\rangle, \quad W_{6}=\left\langle\Delta u_{\mu}\right\rangle\left\langle\chi_{+} u^{\mu}\right\rangle, \quad W_{7}=\left\langle\Delta \chi_{+}\right\rangle\left\langle u_{\mu} u^{\mu}\right\rangle, \\
& W_{8}=\left\langle\Delta u_{\mu} u^{\mu}\right\rangle\left\langle\chi_{+}\right\rangle, \quad W_{11}=\left\langle\Delta \chi_{+}\right\rangle\left\langle\chi_{+}\right\rangle .
\end{aligned}
$$

We have verified that the contribution of the singlet sector to the divergences indeed cancels the terms with a negative power of $N$. It would remain to be checked by an explicit calculation that the inclusion of the fermionic ghosts removes the terms with the linear flavour dependence [13], as one expects.

Finally, we derive the contribution of quenched chiral logs in this sector, according to the simple recipe given in the previous appendix. The answer is very simple: there are no quenched chiral logarithms in the on-shell octet weak sector at one loop. The reason is that if one expands the term proportional to $c_{2}$ in the lowest order Lagrangian around the background field, there are no terms with $\xi_{0}^{2}$. The situation would be different considering also off-shell processes, since the expansion of the $c_{5}$ weak mass term generates the singlet term $c_{5} \xi_{0}^{2}\left\langle\Delta \chi_{+}\right\rangle$. The conclusion here is that the quenched chiral logs can be reabsorbed in a redefinition of $c_{5}$.

\section{One-loop functions}

Here we list the functions $J\left(q^{2}\right), I_{1}\left(q^{2}\right), I_{2}\left(q^{2}\right)$ which appear in the one-loop corrections to the quenched observables analyzed in this paper. $I_{1}\left(q^{2}\right)$ and $I_{2}\left(q^{2}\right)$ are not generated in standard CHPT and arise from the insertion of the $\alpha$ and $m_{0}^{2}$ vertices in any internal singlet line.

The one-loop function $J\left(q^{2}\right)$ in Minkowski space-time, is given by

$$
J\left(q^{2}\right)=\frac{1}{i} \int \frac{d^{d} l}{(2 \pi)^{d}} \frac{1}{\left(M^{2}-l^{2}\right)\left(M^{2}-(l-q)^{2}\right)},
$$

and

$$
J\left(q^{2}\right)=J(0)+\bar{J}\left(q^{2}\right),
$$

where $J(0)$ contains the divergent part

$$
\begin{aligned}
J(0) & =-2 \lambda-\frac{1}{16 \pi^{2}}\left(\ln \frac{M^{2}}{\mu^{2}}+1\right)+O(d-4), \\
\lambda & =\frac{\mu^{d-4}}{16 \pi^{2}}\left[\frac{1}{d-4}-\frac{1}{2}\left(\ln 4 \pi+\Gamma^{\prime}(1)+1\right)\right],
\end{aligned}
$$

and $\bar{J}\left(q^{2}\right)$ is finite. The explicit expression of $\bar{J}\left(q^{2}\right)$ for $d=4$ is:

$$
\bar{J}\left(q^{2}\right)=\frac{1}{16 \pi^{2}}\left\{\sigma \ln \frac{\sigma-1}{\sigma+1}+2\right\},
$$


where $\sigma=\sqrt{1-4 M^{2} / q^{2}}$. The UV finite functions $I_{1}\left(q^{2}\right)$ and $I_{2}\left(q^{2}\right)$ are given by

$$
\begin{aligned}
& I_{1}\left(q^{2}\right)=\frac{1}{i} \int \frac{d^{d} l}{(2 \pi)^{d}} \frac{1}{\left(M^{2}-l^{2}\right)^{2}\left(M^{2}-(l-q)^{2}\right)} \\
& I_{2}\left(q^{2}\right)=\frac{1}{i} \int \frac{d^{d} l}{(2 \pi)^{d}} \frac{1}{\left(M^{2}-l^{2}\right)^{2}\left(M^{2}-(l-q)^{2}\right)^{2}}
\end{aligned}
$$

They are the F.T. of the functions $\tilde{I}_{1}(z)$ and $\tilde{I}_{2}(z)$ :

$$
\begin{aligned}
& I_{1}\left(q^{2}\right)=\frac{1}{i} \int d^{d} z e^{i q z} \tilde{I}_{1}(z), \\
& I_{2}\left(q^{2}\right)=\frac{1}{i} \int d^{d} z e^{i q z} \tilde{I}_{2}(z) .
\end{aligned}
$$

The explicit expression of $I_{1}\left(q^{2}\right)$ and $I_{2}\left(q^{2}\right)$ for $d=4$ is as follows

$$
\begin{aligned}
& I_{1}\left(q^{2}\right)=\frac{1}{16 \pi^{2}} \frac{1}{q^{2}} \frac{1}{\sigma} \ln \frac{\sigma-1}{\sigma+1}, \\
& I_{2}\left(q^{2}\right)=-\frac{1}{8 \pi^{2}} \frac{1}{q^{4} \sigma^{2}}\left[1+\frac{q^{2}-2 M^{2}}{q^{2} \sigma} \ln \frac{\sigma-1}{\sigma+1}\right] .
\end{aligned}
$$

In the text we have used also these functions subtracted at $q^{2}=0$ :

$$
I_{1,2}\left(q^{2}\right)=I_{1,2}(0)+\bar{I}_{1,2}\left(q^{2}\right)
$$

with

$$
I_{1}(0)=\frac{1}{32 \pi^{2} M^{2}}, I_{2}(0)=\frac{1}{96 \pi^{2} M^{4}} .
$$

When $q^{2}>4 M^{2}$ both functions develop an imaginary part, which is given by

$$
\begin{aligned}
\operatorname{Im} I_{1}\left(q^{2}\right) & =\frac{1}{16 \pi} \frac{1}{q^{2}} \frac{1}{\sigma}, \\
\operatorname{Im} I_{2}\left(q^{2}\right) & =-\frac{1}{16 \pi} \frac{2\left(q^{2}-2 M^{2}\right)}{q^{3} \sigma^{3}} .
\end{aligned}
$$

Notice that they diverge at $q^{2}=4 M^{2}$.

\section{References}

[1] A. Morel, J. Phys. (Paris), 48, (1987) 111.

[2] S.R. Sharpe, Phys. Rev. D 41 (1990) 3233, 46 (1992) 3146.

S.R. Sharpe, Nucl. Phys. B (Proc. Suppl.) 30 (1993) 213.

[3] C.W. Bernard, and M.F.L. Golterman, Phys. Rev. D 46 (1992) 853. 
[4] S.R. Sharpe and Y. Zhang, Phys. Rev. D 53 (1996) 5125.

M.J. Booth, Phys. Rev. D 51 (1995) 2338.

[5] M.J. Booth, G. Chiladze and A.F. Falk, Phys. Rev. D 55 (1997) 3092.

[6] J.N. Labrenz and S.R. Sharpe, Phys. Rev. D 54 (1996) 4595; Nucl. Phys. Proc. Suppl. 34 (1994) 335.

G. Chiladze, hep-ph/9704426, prepr. JHU-TIPAC-97008.

[7] M.F.L. Golterman and K.C. Leung, prepr. WASH-U-HEP-97-60, heplat/9702015, to appear in Phys. Rev. D.

[8] J. Gasser, and H. Leutwyler, Ann. of Phys. (N.Y.) 158 (1984) 142.

[9] J. Gasser, and H. Leutwyler, Nucl. Phys. B250 (1985) 465.

[10] G. Colangelo and E. Pallante, Phys. Lett. B 409 (1997) 455.

[11] S. Weinberg, Physica A 96 (1979) 327.

[12] C.W. Bernard, and M.F.L. Golterman, Phys. Rev. D 53 (1996) 476.

[13] G. Colangelo and E. Pallante, work in progress.

[14] J. Gasser, M.E. Sainio and A. Švarc, Nucl. Phys. B 307 (1988) 779.

[15] R. P. Feynman, Phys. Rev. 56 (1939) 340,

H. Hellman, "Einführung in die Quantenchemie", Deuticke, Leipzig, 1937,

S. T. Epstein, Amer. J. Phys. 22 (1954) 613.

[16] M. Ademollo and R. Gatto Phys. Rev. Lett. 13 (1964) 264,

J. Gasser, and H. Leutwyler, Nucl. Phys. B250, (1985) 517.

[17] M. Fukugita, Y. Kuramashi, M. Okawa, H. Mino, and A. Ukawa, Phys. Rev. Lett. 71 (1993) 2387, and Phys. Rev. D 52 (1995) 3003.

[18] C. Bernard et al., Nucl. Phys. B (Proc. Suppl.) 34 (1994) 334.

[19] M. Lüscher, Comm. Math. Phys. 105 (1986) 153.

[20] J. Gasser, and H. Leutwyler, Phys. Lett. 125B (1983) 325.

[21] S.R. Sharpe, Nucl. Phys. B Proc. Suppl. 53 (1997) 181 (LATTICE96).

[22] J. Bijnens, G. Colangelo, G. Ecker, J. Gasser and M. Sainio, Phys. Lett. B 374 (1996) 210.

[23] G. Colangelo, Phys. Lett. B 395 (1997) 389. 
[24] J. Kambor, J. Missimer and D. Wyler, Nucl. Phys. B 346 (1990) 17.

[25] G. Ecker, J. Kambor and D. Wyler, Nucl. Phys. B 394 (1993) 101. 